\title{
Exponential Order Statistic Models of Software Reliability Growth
}

Douglas R. Miller

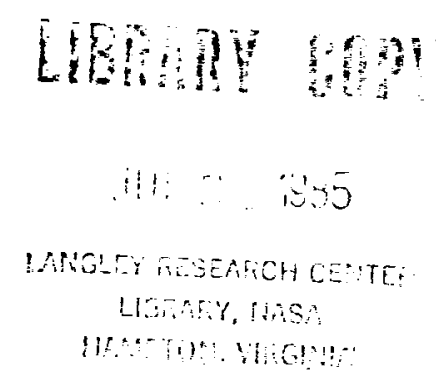



NASA Contractor Report 3909

\section{Exponential Order Statistic Models of Software Reliability Growth}

Douglas R. Miller

The George Washington University

Institute for Management Science and Engineering Washington, D.C.

Prepared for

Langley Research Center under Grant NAG1-179

\section{N/SA \\ National Aeronautics \\ and Space Administration \\ Scientific and Technical Information Branch}



TABLE OF CONTENTS

Page

1. Introduction $\ldots \ldots \ldots \ldots \ldots \ldots \ldots \ldots \ldots \ldots \ldots \ldots \ldots$

2. Exponential Order Statistic Models $\ldots \ldots \ldots \ldots \ldots . . . .3$

3. Doubly Stochastic Exponential Order Statistic Models $\quad \ldots \ldots \ldots \ldots \ldots \ldots \ldots \ldots \ldots \ldots .6$

4. Replication, Nondistinguishability and Approximation ..................... 15

5. Gamma Exponential Order Statistic Models ........ 25

6. Complete Monotonicity Property $\ldots \ldots \ldots \ldots \ldots \ldots \ldots . \ldots 4$

7. Additional Eos Models and Properties .......... 50

8. Inference and Prediction $\ldots \ldots \ldots \ldots \ldots \ldots \ldots \ldots \ldots$

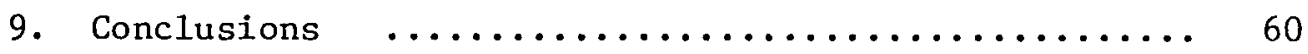



Acknowledgment $\quad \ldots \ldots \ldots \ldots \ldots \ldots \ldots \ldots \ldots \ldots \ldots \ldots$

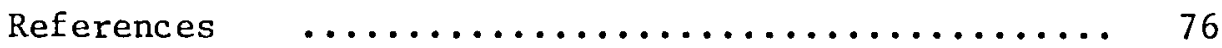



THE GEORGE WASHINGTON UNIVERSITY

School of Engineering and Applied Science

Washington, DC 20052

Institute for Management Science and Engineering

EXPONENTIAL ORDER STATISTIC MODELS

OF SOFTWARE RELIABILITY GROWTH

by

Douglas R. Miller

GWU/IMSE/Seria1 T-496/84

1. Introduction

A system contains design flaws, each of which eventually manifests itself at some point in time, whereupon the system is redesigned in order to remove the design flaw. Design flaws are often called "bugs," and the time points mentioned above will be called "failure times." If the

failure times are indexed chronologically they can be represented as:

$$
0 \leq s_{1} \leq s_{2} \leq s_{3} \leq \cdots
$$

It is convenient to consider (1.1) as the realization of a random process:

$$
0 \leq \mathrm{s}_{1} \leq \mathrm{s}_{2} \leq \mathrm{S}_{3} \leq \cdots,
$$

where the $s_{i}$ 's are random variables and the $s_{i}$ 's are real scalars. If system redesigns successfully remove design flaws, system reliability 
will improve and (1.1) and (1.2) should show a general pattern of increasing interfailure times, i.e., reliability growth. This paper presents and investigates a family of probability models for the failure-time process in (1.2). They are reliability growth models called Exponential order Statistic models.

Exponential Order Statistic (EOS) models are based on the following assumptions: Each design flaw has a rate of occurrence; a flaw causes failures to occur according to a Poisson process if it is not removed; and design flaws cause failures independently of one another. If each flaw is removed the first time it causes a failure, the overall failure-time process corresponds to order statistics from independent but not necessarily identically distributed Exponential random variables. Campbell and ott [4] use such a model. The model is implicit in Nagel's [21,22] replicated software debugging experiments. Adams [1] studies the rates at which design flaws cause failures.

Three different subfamilies of EOS models are considered. These are based on three classes of models for the failure rates of the design flaws initially present in the system: (i) a collection of deterministic rates, (ii) a finite set of random independent identically distributed rates, (iii) a set of random rates whose joint distribution is that of a nonhomogeneous Poisson Process (NHPP). Models of type (i) are deterministic EOS models (denoted DET/EOS), models of types (ii) and (iii) are doubly stochastic EOS models (denoted DS/EOS). Various properties of and interrelationships between these three families are given.

The ultimate goal when developing reliability growth models is the development of good reliability inference and prediction methods which are applied to software development and maintenance. Nevertheless, this paper will not deal with inference or prediction per se. The focus is on the probability models and their properties. However, some of these properties do provide bases for new approaches to estimation and prediction of reliability growth.

The general content of the paper is as follows: Section 2 contains definitions, basic properties and some examples of EOS models with 
deterministic failure rates. Section 3 introduces doubly-stochastic EOS models and tries to portray them as natural extensions of the DET/EOS models. Section 4 presents some general mathematical results as well as some guidelines dealing with problems in distinguishing different models from each other. Section 5 presents an important parametric family of DS/EOS models: the Gamma/EOS mode1s; the closure of this family includes, as special cases, the Jelinsky-Moranda [12] model, the Goel-okumoto [10] mode1, the Littlewood [16] model, the Musa-Okumoto [20] Logarithmic Poisson model, and the Power Law Mode1 [6,7]. In Section 6 the mean function and intensity of the failure-time process (1.2) are characterized using the property of complete monotonicity; this will be useful for nonparametric analyses. Section 7 includes more parametric families of EOS models and additional properties. Section 8 contains some brief comments on possible approaches to inference and prediction using the results of this paper. Conclusions are in Section 9. An Appendix contains proofs and other details omitted from the body of the paper.

\section{Exponential Order Statistic Models}

Let $x_{i}, i=1,2, \ldots, n$, be independent Exponential random variables with rates $\lambda_{i}, i=1,2, \ldots, n$, respectively. (Throughout this paper we assume $0 \leq \lambda_{i}<\infty$.) If $1_{[0, t]}(\cdot)$ is the indicator function of the set $\{x: 0 \leq x \leq t\}$, then

$$
N(t)=\sum_{i=1}^{n} 1_{[0, t]}\left(x_{i}\right), t \geq 0,
$$

equals the number of events occurring by time $t$. Let $s_{i}=\min \{t: N(t) \geq i\}$, $i=1,2, \ldots, n$, be the occurrence time of the $i$ th event, $i . e .$, the $i$ th order statistic of $x_{1}, x_{2}, \ldots, x_{n}$. The stochastic processes $\{N(t), t \geq 0\}$ and $\left\{s_{i}, i=1,2 \ldots\right\}$ are the counting process and the occurrence-time process, respectively, of an Exponential order Statistic (EOS) model with parameter set $\lambda_{\sim}=\left\{\lambda_{1}, \lambda_{2}, \ldots, \lambda_{n}\right\}$. Anticipating the introduction of 
doubly stochastic models in the next section, we shall denote the above model as a deterministic EOS model, abbreviated as DET/EOS.

It is mathematically possible and convenient to permit an infinite number of events, $i . e ., n=\infty$. In this case the parameter set $\underset{\sim}{\lambda}$ is infinite. If $\sum_{i=1}^{\infty} \lambda_{i}<\infty$, then $P(N(t)<\infty)=1$ for $0 \leq t<\infty$, so it is a proper model. If $\sum_{i=1}^{\infty} \lambda_{i}=\infty$, then $\mathrm{P}(\mathrm{N}(\mathrm{t})=\infty)=1$ for $0<t<\infty$, a degenerate situation. (Proofs are given in the Appendix.) The infinite event model can be thought of either as a model of physical reality or as a mathematically tractible approximate model when there is a large but finite number of events. By setting $\lambda_{i}=0, i \geq n+1$, the finite event model is obtained as a special case of the infinite event model. Thus, without loss of generality, infinite event models can be considered. (See, also, Theorem 4.9.)

An Exponential order statistic model is characterized by its parameter set $\lambda_{\sim}^{\lambda}=\left\{\lambda_{i}, \lambda_{2}, \ldots\right\}$. The only restrictions on $\lambda_{\sim}$ are $\lambda_{i} \geq 0, i=1,2, \ldots$, and $\sum_{i=1} \lambda_{i}<\infty$. So the allowable parameter sets for DET/EOS models include many, many possibilities. Some interesting special cases are:

1. $\lambda_{i}=\lambda_{0}, 1 \leq i \leq \mathrm{n}$ (constant rates);

2. $\lambda_{i}=\xi \log (\nu / i), 1 \leq i<[\nu]$ (logarithmic rates);

3. $\lambda_{i}=\alpha \beta^{i}, 1 \leq i<\infty, 0<\beta<1$ (geometric rates);

4. $\lambda_{i}=\xi i^{-\eta}, 1 \leq i<\infty, 1<\eta<\infty$ (power rates).

Case 1 is the famous Jelinsky-Moranda [12] model. Case 2 is another finite event model. Case 3 was observed by Nagel [21,22] for rates 
estimated during replicated-run software debugging experiments. Case 4 follows a pattern observed by $R$. W. Phillips of IBM which is reported by Adams [1]: "the relative numbers of design errors having each possible rate was proportional to a particular inverse power of rates." (This relationship will be revealed more clearly in Section 7.)

A parameter set $\lambda_{\sim}$ can be characterized by a cumulative function: Let $L(\lambda)$ equal the number of elements in $\left\{\lambda_{i} \varepsilon \lambda_{\sim}: \lambda_{i}<\lambda\right\}, 0 \leq \lambda<\infty$. If $\lambda_{\sim}$ is an infinite set with $\sum_{i=1}^{\infty} \lambda_{i}<\infty$, then $L(\lambda)=\infty$ and it will be better to work with the complementary set: Let $\bar{L}(\lambda)$ equal the number of elements in $\left\{\lambda_{i} \varepsilon \lambda_{\nu}: \lambda_{i} \geq \lambda\right\}, 0 \leq \lambda<\infty$. For the above four examples, the (complementary) cumulative functions are:
1. (constant)$$
\overline{\mathrm{L}}(\lambda)= \begin{cases}\mathrm{n}, & 0 \leq \lambda \leq \lambda_{0} \\ 0, & \lambda_{0}<\lambda<\infty\end{cases}
$$
2. (logarithmic)
$\bar{L}(\lambda)=[\nu \exp (-\lambda / \xi)], 0 \leq \lambda<\infty$
3. (geometric)
$\bar{L}(\lambda)=\left\{\begin{array}{cl}{[(\log \lambda-\log \alpha) / \log \beta]} & , 0 \leq \lambda \leq \alpha \\ 0 & , \alpha<\lambda<\infty\end{array}\right.$
4. (power)
$\overline{\mathrm{L}}(\lambda)=\left[(\xi / \lambda)^{1 / \eta}\right], \quad 0 \leq \lambda<\infty$

where $[x]=\max \{i:$ integer, $i \leq x\}$ is the greatest integer function. Note that $\sum_{i=1}^{\infty} \lambda_{i}=\int_{0}^{\infty} \overline{\mathrm{L}}(\lambda) \mathrm{d} \lambda$; thus the set of permissible cumulative functions is precisely the set of all integrable nonnegative decreasing step-functions with integer steps. Deterministic EOS models exist in one-to-one correspondence with these functions. This characterization will be extended in Section 3 to obtain a broader class of EOS models.

The mean function, $M(\cdot)$, of the counting process $\{N(t), 0 \leq t\}$ is defined as $M(t)=E N(t), 0 \leq t$, and using the infinite analogue of (2.1), 


$$
\begin{aligned}
M(t) & =E\left(\sum_{i=1}^{\infty} 1_{[0, t]}\left(x_{i}\right)\right) \\
& =\sum_{i=1}^{\infty} E\left(1_{[0, t]}\left(x_{i}\right)\right) \\
& =\sum_{i=1}^{\infty} P\left(x_{i} \leq t\right) \\
& =\sum_{i=1}^{\infty}\left(1-e^{-\lambda{ }_{i}}\right) \\
& =\int_{0}^{\infty}\left(1-e^{-\lambda t}\right)(-d \bar{L}(\lambda))
\end{aligned}
$$

The intensity function, $\mathrm{m}(\cdot)$, of the counting process is the derivative of the mean function:

$$
\begin{aligned}
\mathrm{m}(\mathrm{t}) & =\mathrm{M}^{i}(\mathrm{t}) \\
& =\sum_{i=1}^{\infty} \lambda_{i} \mathrm{e}^{-\lambda_{i} t} \\
& =\int_{0}^{\infty} \lambda_{\mathrm{e}}{ }^{-\lambda t}(-\mathrm{d} \overline{\mathrm{L}}(\lambda)) .
\end{aligned}
$$

Note that $m(\cdot)$ is the Laplace transform of a measure with masses $\lambda_{i}$ at points $\lambda_{i}, i=1,2, \ldots ;$ the total mass is $\sum_{i=1} \lambda_{i}$, which is finite. From uniqueness of the Laplace transform for probability distributions (see Feller [9, p. 430]), it follows that a DET/EOS model is completely determined by its intensity function $\mathrm{m}(\cdot)$, or equivalently by its mean function $\mathrm{M}(\cdot)$. Of the four previous examples of DET/EOS models, only the first (constant rate) has a mean function with a nice closed form: $M(t)=n\left(1-\exp \left(-\lambda_{0} t\right)\right)$. In effect, this gives an alternative definition of the Jelinsky-Moranda model; It is a DET/EOS model with mean function 
$M(t)=n\left(1-\exp \left(-\lambda_{0} t\right)\right)$. Or, equivalently the $J-M$ model is defined as a DET/EOS model with intensity function $m(t)=n \lambda_{0} \exp \left(-\lambda_{0} t\right)$. The reason for focusing on the mean and intensity functions will become obvious in Sections 3 and 4.

The EOS model is based on two basic assumptions: independence of times until occurrence of different events and Exponential distribution of these times. In the general context of software reliability growth these assumptions will not be exactly met; it can only be hoped that they are reasonable approximations. Independence can fail because of interaction between bugs; for example in experiments performed at the NASA AIRLAB facility, G. E. Migneault has observed instances of interaction, e.g., a program containing two bugs can have a higher failure rate than the sum of the failure rates of the two corrected versions each of which contains only one bug. Exponentiality can fail when the program input changes nonrandomly over time or exhibits a "memory."

There exist several possible justifications for assuming Exponential distributions. In a carefully designed system occurrences of design flaws should be low probability events, i.e., "rare events." Poisson processes with their exponential waiting times are of ten good models for this type of phenomenon; see Breiman [3] and Ross [23]. Campbell and ott [4] use EOS models for manifestation times of design flaws in nuclear reactors. A more rigorous justification for exponentiality can be given for programs that perform calculations based on individual discrete inputs; if a design flaw manifests itself for proportion $p$ of the possible inputs and the successive inputs are chosen independently and randomly, the number of inputs until manifestation will have a Geometric distribution with parameter p. If execution times for each input are independent of success or failure of the computation and $p$ is small, the Exponential is a good approximation (see Appendix). The Exponential will also be a good approximation for this type of program when successive inputs are not independent but are a regenerative process, e.g., successive inputs may correspond to a random walk through the space of all possible program inputs (see Appendix). 
Exponential Order Statistic models based on specific parameter sets are quite useful for specific applications: The Jelinsky-Moranda model is widely used; Migneault (experiments performed at NASA Langley Research Center's AIRLAB facility) has used the EOS model with geometric rates to predict successfully the occurrence of new, as yet unobserved, bugs. It is obviously a rich and useful class of models. However, it seems clear that parameter sets whose elements satisfy exact functional relationships as in the four examples (constant, logarithmic, geometric, or power relationships) can only be approximate. Thus, arbitrary parameter sets should be considered; this leads to nonparametric approaches: Campbell and ott [4], for example. Also, stochastic parameter sets should be considered; this leads to alternative derivations of some existing software reliability growth models and additional insights.

\section{Doubly Stochastic Exponential Order Statistic Models}

Exponential Order Statistic models are based on the assumption that a given bug in a given program has a particular rate of occurrence, $\lambda$. If the program is executed in a time-homogeneous environment the observed rate at which the bug causes errors will converge to $\lambda$ as execution time increases to infinity. By performing replicated-run software debugging experiments $[21,22]$ the error rate for a particular bug can be estimated to any desired degree of accuracy. In this sense, for a given program in a time-homogeneous usage environment the parameter set $\lambda=\left\{\lambda_{1}, \lambda_{2}, \ldots\right\}$ is a set of deterministic values.

Now let use consider possible models for the parameter set $\lambda$. In the previous section, various deterministic functional relationships were presented. If design errors are accidentally included in program, it is difficult to imagine that their rate would obey exact functional relationships. It is more plausible that the rates would be described by these relationships plus some random noise. This suggests that $\underset{\sim}{\lambda}$ be modeled as a realization of a stochastic point process; for example $\left\{\lambda_{1}, \lambda_{2}, \ldots\right\}$ could be a realization of a nonhomogeneous Poisson process (NHPP). This gives a doubly-stochastic model: one process for the rates, and a DET/EOS process for occurrence times conditional on the 
rates. It is a doubly-stochastic exponential order statistic (DS/EOS) mode1.

Define $\Lambda_{\imath}=\left(\Lambda_{1}, \Lambda_{2}, \Lambda_{3}, \ldots\right)$ as a point process on $(0, \infty)$, i.e., a collection of random points greater than 0 . We shall consider $\Lambda$ as a random perturbation from a deterministic model: Let $\bar{L}(\lambda), 0<\lambda$, be any nonincreasing function which decreases to 0 as $\lambda$ increases. Define $\lambda_{i}=\bar{L}^{-1}(i), i=1,2,3, \ldots$; for example, $\bar{L}(\lambda)=\nu \exp (-\lambda / \xi), 0<\lambda$, gives the logarithmic deterministic set of rates from the previous section. From Figure 3.1 we see that a random perturbation from this deterministic model can be achieved by perturbing $\{1,2, \ldots n\}$ into the new values $\left\{u_{1}, u_{2}, \ldots u_{n}\right\}$ and then defining, $\lambda_{i}=\bar{L}^{-1}\left(u_{i}\right), i=1,2, \ldots n$. The set $\left\{u_{i}, u_{2}, \ldots, u_{n}\right\}$ can be the realization of any point process $\mathrm{U}=\left(\mathrm{U}_{1}, \mathrm{U}_{2}, \ldots\right)$ on $(0, v)$, but we shall only consider two possibilities: (a) $U$ is the process of $n$ independent Uniform $[0, v]$ order statistics, and (b) $U$ U is a homogeneous Poisson process (HPP) with unit rate. For these cases we have the following general theorems (see Appendix for proofs):

Theorem 3.1: Let $\overline{\mathrm{L}}(\lambda)$ be a nonincreasing function on $[0, \infty)$ such that $\overline{\mathrm{L}}(0)=\mathrm{n}$ and $\lim _{\lambda \rightarrow \infty} \overline{\mathrm{L}}(\lambda)=0$. Let $\mathrm{v}_{\nu}^{\mathrm{U}}=\left(\mathrm{U}_{1}, \mathrm{U}_{2}, \ldots, \mathrm{U}_{\mathrm{n}}\right)$ be $n$ independent Uniform $[0, n]$ order statistics. Define $\Lambda_{i}=\bar{L}^{-1}\left(U_{i}\right)$, $i=1,2, \ldots, n$. Then $\Lambda_{n}=\left(\Lambda_{1}, \Lambda_{2}, \ldots, \Lambda_{n}\right)$ are $n$ independent order statistics from a distribution with complementary cdf $\overline{\mathrm{L}}(\cdot) / \mathrm{n}$ and complementary cumulative mean function $\overline{\mathrm{L}}(\cdot)$.

Theorem 3.2: Let $\overline{\mathrm{L}}(\lambda)$ be a nonincreasing function on $[0, \infty)$ such that $\lim _{\lambda \rightarrow \infty} \overline{\mathrm{L}}(\lambda)=0$. Let $\underset{\sim}{\mathrm{U}}=\left(\mathrm{U}_{1}, \mathrm{U}_{2}, \ldots\right)$ be a homogeneous Poisson process with rate 1 . Define $\Lambda_{i}=\bar{L}^{-1}\left(U_{i}\right)$ for $i$ such that $\mathrm{U}_{i}<\overline{\mathrm{L}}(0)$. Then $\Lambda_{\sim}=\left(\Lambda_{1}, \Lambda_{2} \ldots\right)$ is a nonhomogeneous Poisson process with complementary cumulative mean function $\overline{\mathrm{L}}(\cdot)$. 


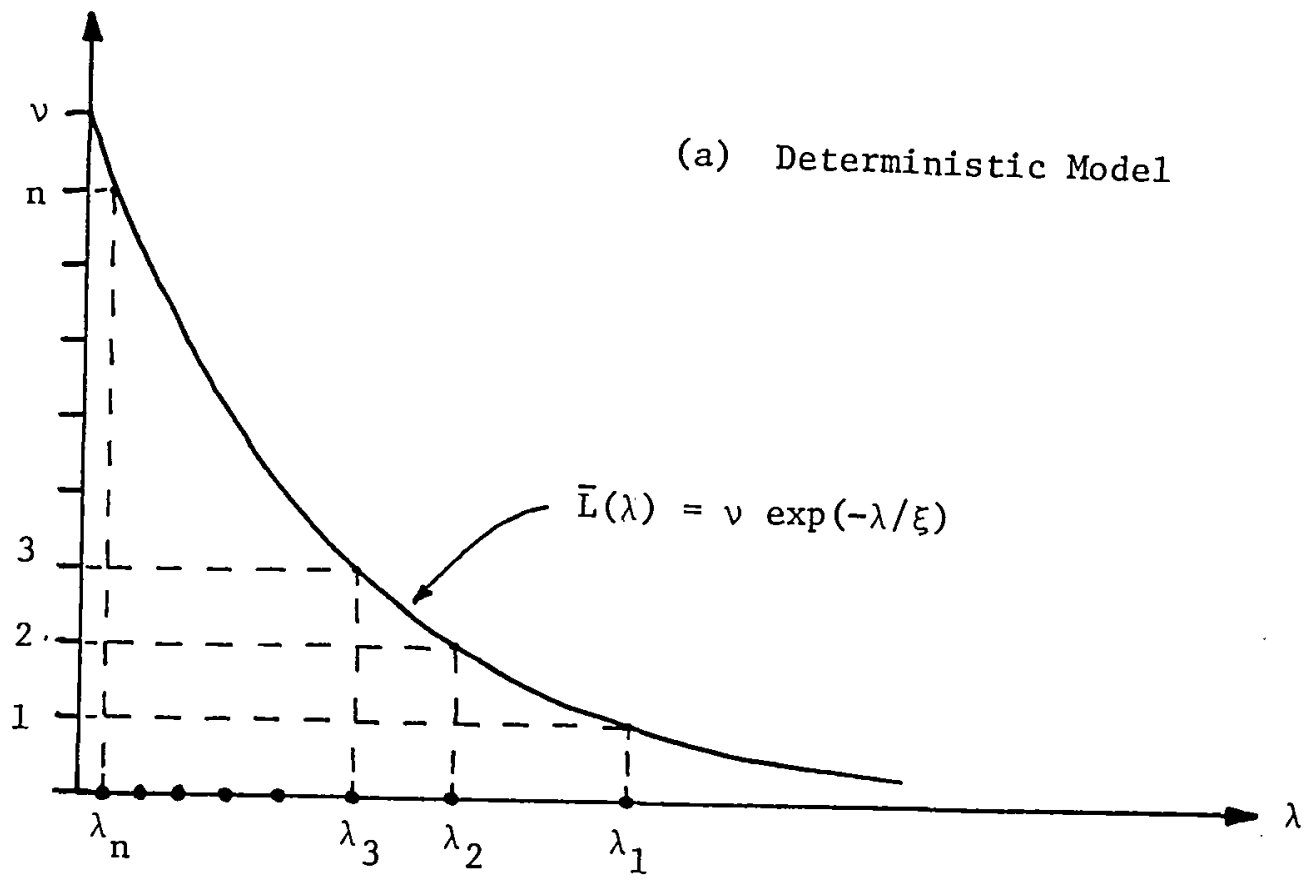

$$
\lambda_{i}=\xi(\log v-\log i), i=1,2, \ldots, n
$$

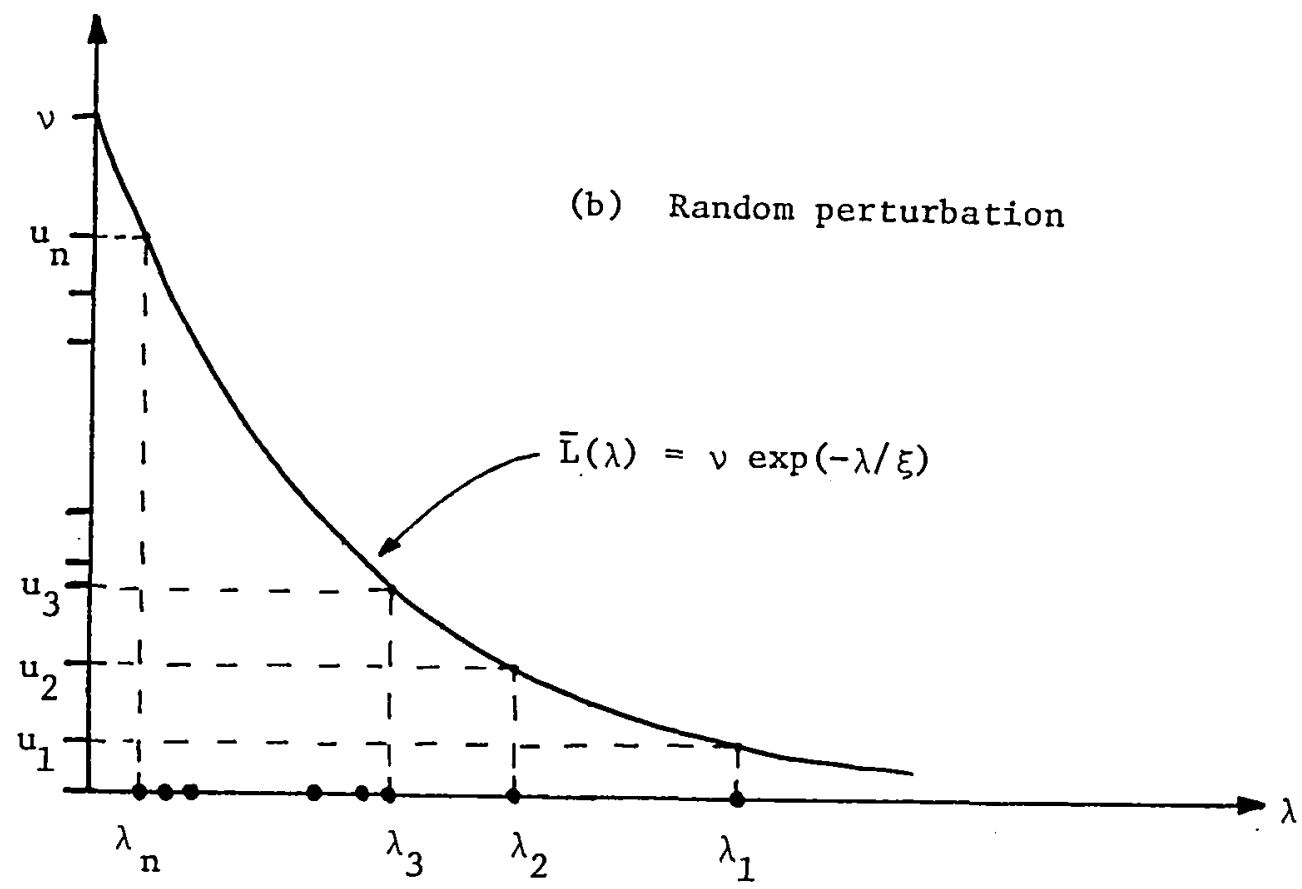

Figure 3.1 Deterministic model and random perturbation model of rates 
Thus for a given function $\overline{\mathrm{L}}(\cdot)$ with $\overline{\mathrm{L}}(0)<\infty$, we consider three models for the rates $\left\{\lambda_{1}, \lambda_{2}, \ldots\right\}:$ (a) i.i.d. order statistics from $1-\overline{\mathrm{L}}(\cdot) / \overline{\mathrm{L}}(0)$; (b) NHPP with mean function $\overline{\mathrm{L}}(0)-\overline{\mathrm{L}}(\cdot)$; and (c) deterministic model. If $\overline{\mathrm{L}}(0)=\infty$, only cases (b) and (c) are possible and the NHPP must be described using the complementary mean function $\bar{L}(\cdot)$. It is hoped that looking upon these random models as perturbations of the deterministic models will provide some motivation for their consideration. Another approach would be simply to postulate the random models without mentioning the associated deterministic model. Regardless, they will be useful and interesting.

The Jelinsky-Moranda mode1 (constant rates) has $\overline{\mathrm{L}}(\lambda)=$ $\mathrm{n} 1_{\left[\lambda_{0}, \infty\right)}(\lambda), 0 \leq \lambda$. If $\overline{\mathrm{L}}$ is approximated by a smooth curve such as the complementary $\mathrm{cdf}, \overline{\mathrm{G}}(\cdot)$, of a Gamma distribution (with mean $\lambda_{0}$ and large shape parameter) multiplied by $\mathrm{n}$, we get a "noisy" J-M model; see Figure 3.2 . Following the above there are two options: $\Lambda$ may be (a) $n$ i.i.d. order statistics from $\bar{G}(\cdot)$, or (b) an NHPP with cumulative mean function $n(1-\bar{G}(\cdot))$. Another simpler possibility for introducing noise into the J-M model is an NHPP with complementary cumulative mean function $\overline{\mathrm{L}}(\lambda)=\mathrm{n} 1_{\left[\lambda_{0}, \infty\right)}(\lambda), 0<\lambda$, i.e., $\mathrm{N}$ rates all equal to $\lambda_{0}$, where $\mathrm{N}$ has a Poisson distribution with mean $\mathrm{n}$.

We are considering i.i.d. order statistic (IIDOS) models and NHPP models for $\Lambda$. Another possible dichotomy among models concerns finite and infinite component $\Lambda$. This is only relevant for NHPP's. It turns out to be unnecessary to make this distinction because NHPP's can be defined generally regardless of whether $\overline{\mathrm{L}}(0)$ is finite or infinite. The transformation of an HPP into an NHPP is illustrated in Figure 3.3 for both cases. Thus we consider IIDOS models and NHPP models, each of the former having a finite deterministic number of bugs and each of the latter having either a finite (Poisson-distributed) number or infinite number of bugs. In all cases $\overline{\mathrm{L}}(\lambda)$ denotes the complementary cumulative mean function, i.e., the expected number of bugs which have rates greater 



Figure 3.2 The complementary cumulative mean function for the rates of J-M model, $\mathrm{L}(\cdot)$, and an approximation based on the gamma distribution 

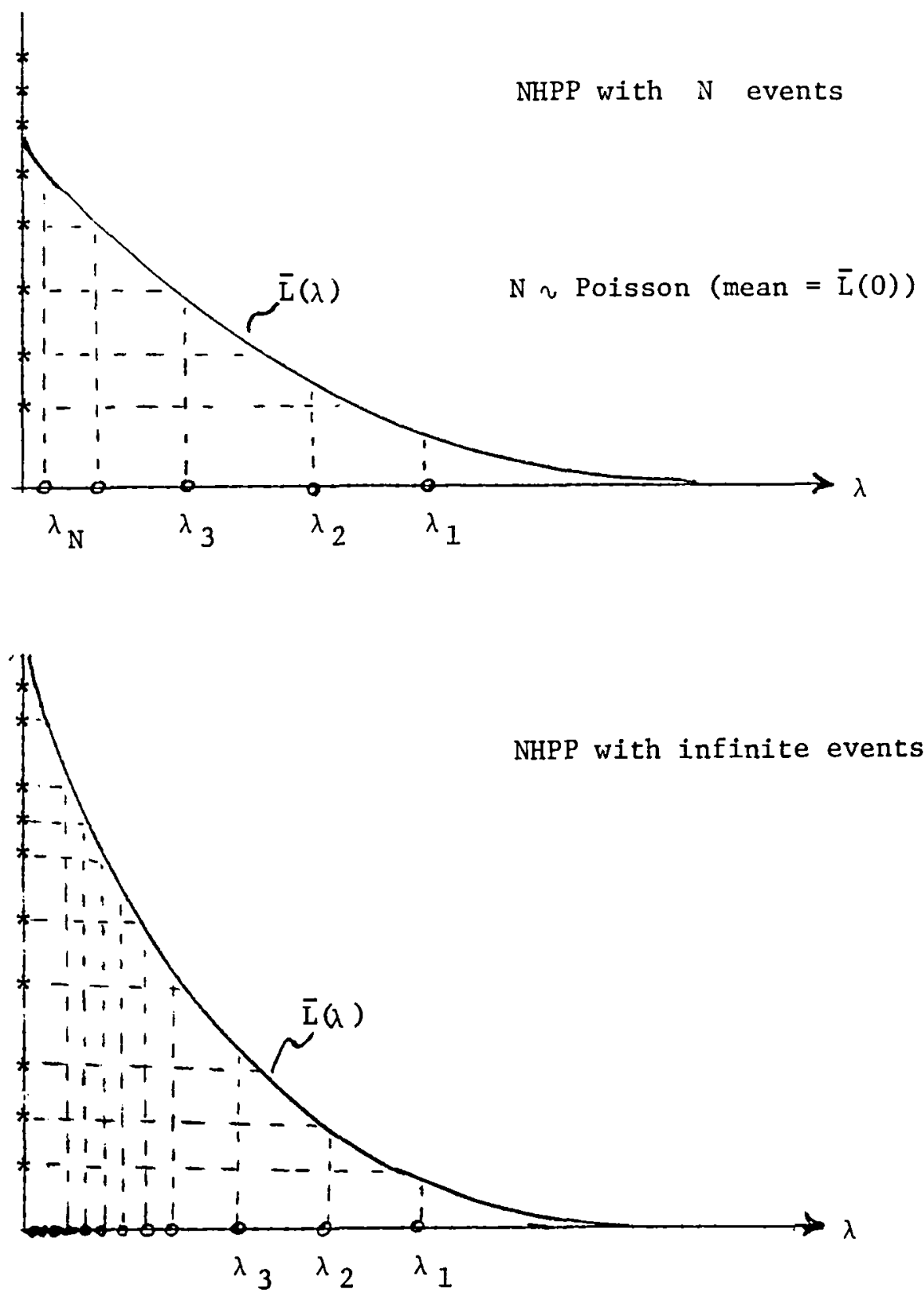

Figure 3.3 Nonhomogeneous Poisson processes: finite and infinite number of events 
than or equal to $\lambda$.

The DS/EOS processes $\{N(t), 0<t\}$ have especially nice forms for IIDOS $\Lambda^{\prime}$ 's and for NHPP $\Lambda^{\prime}$ 's:

Theorem 3.3: Let $\Lambda$ be an IIDOS process with cumulative mean function $L(\lambda), 0 \leq \lambda$. Then, the DS/EOS process with rates $\Lambda$ is an IIDOS process with mean function $M(t)=\int_{0}^{\infty}\left(1-e^{-\lambda t}\right) d L(\lambda), 0 \leq t$, and intensity $m(t)=\int_{0}^{\infty} \lambda \mathrm{e}^{-\lambda \mathrm{t}} \mathrm{dL}(\lambda), 0 \leq \mathrm{t}$.

Theorem 3.4: Let $\Lambda$ be an NHPP process with complementary cumulative mean function $\overline{\mathrm{L}}(\lambda), 0 \leq \lambda$. Then, the DS/EOS process with rates $\Lambda$ is an NHPP with mean function $M(t)=\int_{0}^{\infty}\left(1-e^{-\lambda t}\right)(-d \bar{L}(\lambda))$, $0 \leq t$, and intensity $m(t)=-\int_{0}^{\infty} \lambda e^{-\lambda t} d \bar{L}(\lambda), 0 \leq t$.

Note that the above DS/EOS processes are completely specified by whether $\Lambda$ is IIDOS or NHPP and by the complementary cumulative mean function $\overline{\mathrm{L}}(\cdot)$. They can be denoted as IIDOS/EOS $(\overline{\mathrm{L}})$ and NHPP/EOS $(\bar{L})$, respectively. With this notation the above theorems can be summarized as

$$
\begin{aligned}
& \operatorname{IIDOS} / \operatorname{EOS}(\overline{\mathrm{L}})=\operatorname{IIDOS}(\mathrm{i} 1) \\
& \operatorname{NHPP} / \operatorname{EOS}(\overline{\mathrm{L}})=\operatorname{NHPP}(\mathrm{M}),
\end{aligned}
$$

where $M(t)$ is defined above. Thus by a rather circuitous, but physically meaningful, route we have arrived at some familiar models.

Now consider doubly stochastic versions of the four examples from Section 2:

$$
\begin{aligned}
& \text { 1. } \overline{\mathrm{L}}(\lambda)=\left\{\begin{array}{l}
\mathrm{n}, 0 \leq \lambda \leq \lambda_{0} \\
0, \lambda_{0}<\lambda<\infty
\end{array} \quad ; \mathrm{M}(\mathrm{t})=\mathrm{n}\left(1-\mathrm{e}^{-\lambda_{0} t}\right)\right. \\
& \text { 2. } \overline{\mathrm{L}}(\lambda)=\nu \exp (-\lambda / \xi) \quad ; \mathrm{M}(\mathrm{t})=\nu\left(\frac{\xi \mathrm{t}}{\xi \mathrm{t}+1}\right)
\end{aligned}
$$




$$
\begin{aligned}
& \text { 3. } \overline{\mathrm{L}}(\lambda)=\left(\frac{\log \lambda-\log \alpha}{\log \beta}\right)^{+} ; \mathrm{M}(\mathrm{t})=\frac{1}{\log \beta} \sum_{i=1}^{\infty} \frac{(-\alpha t)^{i}}{i \cdot i !} \\
& \text { 4. } \overline{\mathrm{L}}(\lambda)=(\xi / \lambda)^{1 / n} \quad ; \mathbb{M}(\mathrm{t})=\Gamma(1-1 / \eta)(\xi \mathrm{t})^{1 / n}
\end{aligned}
$$

In case 1 , IIDOS $(M)$ is still the $J-11$ model, but NHPP(M) is the Goelokumoto [10] model. In case 2, for integer $\nu$ IIDOS(M) is a case of the Littlewood [16] model. Cases 3 and 4 are infinite event models and therefore are NHPP's: Case 3 is apparently new. Case 4 is a familiar NHPP, sometimes referred to as the Weibull NHPP because its intensity function is identical to the Weibull hazard rate function; Crow [6] and Duane [7] have used it as a model of reliability growth. Thus DS/EOS models provide alternative derivations for some existing proven reliability growth models.

4. Replication, Nondistinguishability and Approximation

We are studying three families of processes: exponential order statistic (EOS) models based on a deterministic parameter set $\stackrel{\lambda}{\lambda}=\left\{\lambda_{1}, \lambda_{2}, \ldots\right\}$, doubly stochastic exponential order statistic (DS/EOS) models whose parameter sets are order statistics of independent identically distributed (IIDOS) random variables, and DS/EOS models whose parameter sets are nonhomogeneous Poisson processes (NHPP). We are interested in relationships between these families of models. In particular, we seek answers to the questions: What role does replication play? In what senses can these families be distinguished from one another? In what senses can they be used as approximations to one another? Within individual families, do some models approximate others?

The role of replication is important and needs clarification. There are two replication concepts: (a) separate programmers or groups of programmers can independently create different versions of the same program, and (b) a single program may be subjected to several separate debugging runs $[8,21,22]$. Figure 4.1 illustrates the program creation 




Figure 4.1 Different replication concepts 
and running and the two replication possibilities: From a single program specification, the program is created (Box A) and then subjected to a debugging/correction run (Box B). Replicated debugging/correction runs for a single program are represented by Loop B. Replicated program creation corresponds to independent passes through Box A. Replicated creation and a debugging/correction run is represented by Loop A. A nested design would involve several replicated runs for each replicated program, for example in Nagel's [22] original pioneering experiment each program specification had two replicated programs and 50 replicated debugging runs, i.e., two passes through Loop A, each containing 50 passes through Loop $B$.

In this paper we are assuming certain stochastic models for what happens in Box $A$ and Box B of Figure 4.1. In Box A a program is created containing bugs. In Box $B$, the program is run for a certain length of time; when a bug manifests itself it is corrected. It is assumed that the usage environment is time homogeneous throughout the experiment; so we assume that each bug has a fixed (unknown) deterministic manifestation rate associated with it. We assume three possible models in Box A: these rates $\left\{\lambda_{1}, \lambda_{2}, \ldots\right\}$ are the realization of a deterministic process, an IIDOS process, or an NHPP. Given the rates produced in Box A, the manifestation times $\left\{s_{1}, s_{2}, \ldots\right\}$ generated in Box B will be the realization of a DET/EOS process with those rates. Thus, from Section 3 , we see that a single pass through Box $A$ and then Box $B$ leads to one observation of a DET/EOS process, an IIDOS process, or an NHPP; this depends upon whether Box A is deterministic, an IIDOS model, or an NHPP model, respectively. This means that independent replications around Loop A of Figure 4.1 each involving a single run (one pass through Box $B$ for each pass through Box A) will yield independent realizations of either a DET/EOS, IIDOS, or NHPP model. If a large number of replicates is taken, then by standard statistical consistency arguments it is theoretically possible to distinguish between the three models and to estimate the model parameters to any desired degree of accuracy. This is rather unrealistic in practice, however. Existence of a single replicate is probably more common and meaningful when EOS models are used. 
Multiple replications can involve either replicated programs or replicated debugging/correction runs. For replicated versions of the same program it is unreasonable to model the error rates $\left\{\lambda_{1}, \lambda_{2}, \ldots\right\}$ for different programmers as independent, identically distributed realizations of a single simple stochastic process; replicated-run experiments performed to date $[8,21,22]$ suggest a possible noisy geometric pattern (Example 3 of Section 2) for different programmers but with distinctly different parameters. As Nagel [22] points out, there is a definite programmer effect. Thus for replicated programs (Box A of Figure 4.1) more complicated models are needed for $\Lambda$. The IIDOS and NHPP models we use for $\Lambda$ should be considered as a way of introducing noise into the pattern of error rates, not as a model for different programmers. (It might also be interpreted in terms of Bayesian subjective probability for the individual programmer.) If multiple debugging/correction runs are performed for a single version of a program, then we observe replicated sample paths of a DET/EOS process with a given $\lambda_{\sim}^{\lambda}=\left\{\lambda_{1}, \lambda_{2}, \ldots\right\}$. However, with such an experimental design it is more natural and efficient (for estimation of $\lambda$ ) to identify the bug which corresponds to each failure observed. Thus the data will be collected as samples of manifestation times for each bug observed rather than sample paths of a DET/EOS process. This leads to different questions and analyses than those with which this paper is dealing. Thus for the scheme of Figure 4.1, we will avoid replication. Furthermore, and very importantly, most software reliability growth data will consist of a single replicate. Thus we shall have a single replicate of either a DET/EOS model, an IIDOS mode1, or a NHPP model.

Let us consider the problem of distinguishing between an IIDOS model and a NHPP model by observing one realization of the process. Let $F(\cdot)$ be a cdf on $[0, \infty)$ and let $\left\{N_{1}(t), 0 \leq t\right\}$ be the counting process for an IIDOS model with $m$ observations, i.e., cumulative mean function $\mathrm{M}_{1}(\mathrm{t})=\mathrm{mF}(\mathrm{t}), 0 \leq t$, and occurrence times $\left\{\mathrm{s}_{1,1}, \mathrm{~s}_{1,2}, \ldots, \mathrm{s}_{1, \mathrm{~m}}\right\}$. Let $\left\{N_{2}(t), 0 \leq t\right\}$ be the counting process for an NHPP with cumulative mean function $M_{2}(t), 0 \leq t$, and occurrence times $\left\{s_{2,1}, s_{2,2}, s_{2,3}, \ldots\right\}$ 
There are several well-known relevant theorems:

Theorem 4.1: Suppose $M_{2}(t)=\gamma F(t), 0 \leq t$, where $0<\gamma<\infty$. Conditional on $\mathrm{N}_{2}(\infty)=\mathrm{n},\left(\mathrm{S}_{2,1}, \mathrm{~S}_{2,2}, \ldots, \mathrm{s}_{2, \mathrm{n}}\right)$ are distributed as i.i.d. order statistics with $\operatorname{cdf} F(\cdot), i . e .,\left(s_{2,1}, s_{2,2}, \ldots, s_{2, n}\right)$ is an IIDOS process with mean function $\mathrm{nF}(\cdot)$.

Theorem 4.2: Let $\mathrm{T}$ be a deterministic time or a random time independent of $\left\{\mathrm{N}_{2}(t), 0 \leq t\right\}$. Conditional on $\left\{\mathrm{T}=\mathrm{s}, \mathrm{N}_{2}(\mathrm{~s})=\mathrm{n}\right\}$, $\left(s_{2,1}, s_{2,2}, \ldots, s_{2, n}\right)$ are distributed as i.i.d. order statistics with $\operatorname{cdf} G(t)=M_{2}(t) / M_{2}(s), 0 \leq t \leq s$.

Theorem 4.3: Conditional on $\left\{s_{2, n+1}=s\right\},\left(s_{2,1}, s_{2,2}, \ldots, s_{2, n}\right)$ are distributed as i.i.d. order statistics with $\operatorname{cdf} G(t)=M_{2}(t) / M_{2}(s)$, $0 \leq t \leq s$

Theorem 4.4: Let $T$ be a deterministic time or a random time independent of $\left\{\mathrm{N}_{1}(\mathrm{t}), 0 \leq \mathrm{t}\right\}$. Conditional on $\left\{\mathrm{T}=\mathrm{s}, \mathrm{N}_{1}(\mathrm{~s})=\mathrm{n}\right\}$, $\left(\mathrm{S}_{1,1}, \mathrm{~S}_{1,2}, \ldots, \mathrm{S}_{1, \mathrm{n}}\right)$ are distributed as $\mathrm{i} . \mathbf{i} . \mathrm{d}$. order statistics with $\operatorname{cdf} G(t)=F(t) / F(s), 0 \leq t \leq s$.

Theorem 4.5: Conditional on $\left\{s_{1, n+1}=s\right\},\left(s_{1,1}, s_{1,2}, \ldots, s_{1, n}\right)$ are distributed as i.i.d. order statistics with $\operatorname{cdf} G(t)=F(t) / F(s)$, $0 \leq \mathrm{t} \leq \mathrm{s}$

Theorem 4.6: Let $\mathrm{T}_{1}$ be a stopping time for $\left\{\mathrm{N}_{1}(t), 0 \leq t\right\}$ and let $T_{2}$ be the identically defined stopping time for $\left\{\mathrm{N}_{2}(t), 0 \leq t\right\}$. If $M_{2}(t)=\gamma F(t), 0 \leq t \leq s, 0<\gamma<\infty$, then $\left\{N_{1}(t), 0 \leq t \leq s \mid T_{1}=s\right.$, $\left.\mathrm{N}_{1}(\mathrm{~s})=\mathrm{n}\right\}$ and $\left\{\mathrm{N}_{2}(\mathrm{t}), 0 \leq t \leq \mathrm{s} \mid \mathrm{T}_{2}=\mathrm{s}, \mathrm{N}_{2}(\mathrm{~s})=\mathrm{n}\right\}$ are identically distributed. 
The implication of the above theorems is this: If a single replicate of a reliability growth process is observed over $[0, s]$ in such a way that the assumptions of the above theorems are met it will be impossible to distinguish between certain families of IIDOS models and related families of NHPP models. As an example, consider two sets of cumulative mean functions,

$M_{1}=\left\{M_{1}(\cdot): M_{1}(t)=m(1-\exp (-\lambda t)), m=0,1,2, \ldots, 0<\lambda<\infty\right\}$ and $M_{2}=\left\{M_{2}(\cdot): M_{2}(t)=\gamma(1-\exp (-\lambda t)), 0<\gamma<\infty, 0<\lambda<\infty\right\} ;$ and the two hypotheses $\mathrm{H}_{1}: \operatorname{IIDOS}\left(\mathrm{M}_{1} \in M_{1}\right)$ and $\mathrm{H}_{2}: \operatorname{NHPP}\left(M_{2} \varepsilon M_{2}\right)$, which correspond to hypothesizing the J-M models or G-O models respectively, mentioned in Section 3. Because the scale factors ( $m$ for $M_{1}$ and $\gamma$ for $M_{2}$ ) are completely general it is impossible to distinguish between $\mathrm{H}_{1}$ and $\mathrm{H}_{2}$ based on the number of events in $(0, s)$, i.e., a single observation comes from $\mathrm{H}_{1}$ : Binomial or $\mathrm{H}_{2}$ : Poisson; the only possibility is to look at the joint distribution of times of occurrence (which includes whether the stopping rule is satisfied). But, by Theorems 4.2 through 4.5 , for both $\mathrm{H}_{1}$ and $\mathrm{H}_{2}$ the accurrence times are i.i.d. order statistics from $G(t)=(1-\exp (-\lambda t)) /(1-\exp (-\lambda s))$, with unknown $\lambda, 0<\lambda<\infty$; thus we cannot distinguish between $\mathrm{H}_{1}$ and $\mathrm{H}_{2}$ in the classical hypothesis testing sense if only one replicate is available. If a more general stopping rule is used Theorem 4.6 gives an analogous result. This example generalizes to include any family of cdf's, $F=\left\{\mathrm{F}_{\alpha}: \alpha \varepsilon \mathrm{A}\right\}:$ let $M_{1}=\left\{\mathrm{H}_{1}(\cdot): \mathbb{M}_{1}(\mathrm{t})=\mathrm{mF}_{\alpha}(\mathrm{t}), \mathrm{m}=0,1,2, \ldots, \alpha \varepsilon \mathrm{A}\right\}$ and $M_{2}=\left\{M_{2}(\cdot): \mathbb{H}_{2}(t)=\gamma F_{\alpha}(t), 0<\gamma<\infty, \alpha \varepsilon A\right\}$, then $\mathrm{H}_{1}: \operatorname{IIDOS}\left(\mathrm{M}_{1} \in M_{1}\right)$ and $\mathrm{H}_{2}: \operatorname{NHPP}\left(\mathrm{M}_{2} \in M_{2}\right)$ cannot be distinguished by observing one replicate. 
This nondistinguishability between families of IIDOS models and NHPP models is really equivalent to an important fact first noted by Langberg and Singpurwalla [14] within the context of reliability growth modeling: Theorem 4.1 has the interpretation that any NHPP with $M(\infty)<\infty$ is a mixture of IIDOS processes with a Poisson [mean $I(\infty)$ ] number of observations from cdf $F(t)=M(t) / M(\infty)$. The above nondistinguishability is thus equivalent to the problem of distinguishing between a constant population and a Poisson population with totally unknown mean when only one observation is available. Of course additional information may destroy the nondistinguishability: for example, bounds on the number of order statistics or the mean of the Poisson distribution may enable conclusions to be drawn from one observation. However, the situation arises frequently where parametric models are postulated with no constraints or information assumed for parameter values. (Theorem 4.1 also plays an additional unifying role if the distribution of $\mathrm{N}(\infty)$ is considered subjectively for a Bayesian analysis; see Langberg and Singpurwalla [14].)

In a weaker sense it is impossible to distinguish between deterministic and doubly stochastic EOS processes using a single replicate. For a given program it is possible to estimate the parameter set $\lambda=\left\{\lambda_{1}, \lambda_{2}, \ldots\right\}$ to any desired accuracy by replicated debugging (Loop B of Figure 4.1). However, even if the parameter set is completely known it still is a single replicate of the $\Lambda$-process and we are again faced with the problem of choosing between a deterministic model and a stochastic model when only one observation is available. If all possible deterministic models and a11 possible IIDOS and NHPP models for $\Lambda$ are allowed then there is no statistical basis for choosing from one class rather than the other. If the choice is restricted to certain families of models, e.g., parametric families of deterministic or stochastic models of $\Lambda$, then it may be possible to distinguish between deterministic and stochastic.

Now consider the very restrictive case where the cumulative mean function $L(\cdot)$ of the $\Lambda$-process is known, but the $\Lambda$-process may be 
either deterministic or an IIDOS process; i.e., let $\mathrm{L}_{0}(\cdot)$ be a known function such that $\mathrm{L}_{0}(\infty)=\mathrm{n}+1, \Lambda_{\sim}$ either is $\left\{\lambda_{i}=\mathrm{L}_{0}^{-1}(i), i=1,2, \ldots, n\right\}$ or is an IIDOS of $n$ observations from $F_{0}(\cdot)=L_{0}(\cdot) /(n+1)$. Note that the deterministic model is equivalent to sampling without replacement from a population with $\operatorname{cdf} F_{1}(\cdot)=\left[(n+1) F_{0}(\cdot)\right] / n$. Note that, if $\mathrm{n}$ is moderately large, $\mathrm{F}_{0}$ and $\mathrm{F}_{1}$ are very close to each other. From a single observation of the failure process $\{N(t), 0 \leq t\}$, it will be virtually impossible to determine whether $\lambda$ 's are drawn with replication from $F_{0}$ or $F_{1}$. However, there will be some measure of distinguishability based on sampling with replacement (from $\mathrm{F}_{0}$ ) or without replacement (from $F_{1}$ ): both processes will fluctuate about the mean $M_{0}(t)=\int_{0}^{\infty}(1-\exp (-\lambda t)) d L_{0}(\lambda)$, but there will be less variability in the process without replacement (the deterministic EOS process). Thus in principle, a statistical test could be based on variation about $\mathrm{M}_{0}(\cdot)$ : for small variation the deterministic model is preferred and for large, the IIDOS model. It is hard to imagine that such a test would have much power. Finally, a more realistic scenario would include a large parametric family (not just one function), and furthermore the single replicate of the failure process would be observed only for a finite time interval, not in its entirety. This makes matters even worse and leads one to the conclusion that, in a practical sense, deterministic EOS processes and IIDOS/EOS processes that have a moderately large number of events and are based on the same parametric family of $L(\cdot)^{\prime}$ 's cannot be distinguished by observing a single replicate of the failure process.

There are some theorems relevant to this discussion. Let $\overline{\mathrm{L}}(\cdot)$ be a function that decreases to 0 on $(0, \infty)$ such that $M(t)=-\int(1-\exp (-\lambda t)) d \bar{L}(\lambda)$ exists for $0 \leq t<\infty$. Let $\left\{\mathrm{N}_{0, k}(\mathrm{t}), 0 \leq \mathrm{t}\right\}, \mathrm{k}=1,2, \ldots$, be a sequence of independent deterministic EOS processes; the parameter set of the $k$ th process is 
$\left\{\lambda_{i}=\bar{L}^{-1}(i / k), i=1,2, \ldots\right\}$. If $\overline{\mathrm{L}}(0)<\infty$, let $\left\{N_{1, k}(t), 0 \leq t\right\}$

be a sequence of independent IIDOS/EOS processes; the parameter set of the $k$ th process consists of $[k \bar{L}(0)] i . i . d$. order statistics from cdf $F(\lambda)=\left(\overline{\mathrm{L}}(0)-\overline{\mathrm{L}}(\lambda) / \overline{\mathrm{L}}(0)\right.$. Let $\left\{\mathrm{N}_{2, \mathrm{k}}(\mathrm{t}), 0 \leq \mathrm{t}\right\}$ be a sequence of independent NHPP/EOS processes; the parameter set of the $k$ th process is an NHPP with complementary mean function $k \bar{L}(\cdot)$.

Theorem 4.7: For $\mathrm{j}=0,1$, and 2,

$$
P\left\{\lim _{k \rightarrow \infty} k^{-1} N_{j, k}(t)=M(t), 0 \leq t<\infty\right\}=1 .
$$

Theorem 4.8: For $j=0,1$, and $\left.2,\left\{N_{j, k}(t)-M(t)\right) / \sqrt{k}, 0 \leq t\right\}$ converge in distribution to Gaussian processes as $k \rightarrow \infty$.

(The covariance structures of the limiting processes are different for $j=0,1$, and 2; see Appendix.)

These theorems show the central role played by the mean function $M(\cdot)$ for processes with a large number of events, regardless of the particular type of process. This plus the previously mentioned problems in distinguishing between the three types of models suggests focusing on the mean function. Thus it might be useful to think of the mean function as the primary characteristic of the model and the particular type of model (DET/EOS, IIDOS, or NHPP) as a secondary characteristic. This is not a new idea; Musa and Okumoto [20] "include" the Littlewood-Verrall model in their study by means of an NHPP with the appropriate mean function.

We shall consider two models to be "close" to each other if their respective mean functions are close. The following limit theorems make this more precise. 


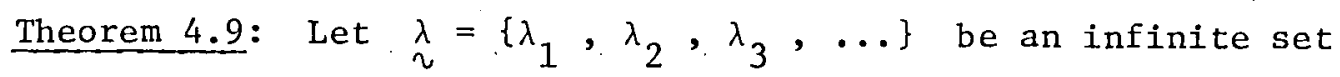
of rates such that $\sum_{i=1}^{\infty} \lambda_{i}<\infty$. Let $\left\{N_{0, k}(t), 0 \leq t\right\}$ be a DET/EOS process with mean function $M_{k}(t)=\sum_{i=1}^{k}\left(1-\exp \left(-\lambda_{i} t\right)\right), 0 \leq t<\infty$, $k=1,2, \ldots$. Then, as $k \rightarrow \infty,\left\{N_{0, k}(t), 0 \leq t\right\}$ converges in distribution to a DET/EOS process with mean function $M_{\infty}(t)=\sum_{i=1}^{\infty}\left(1-\exp \left(-\lambda_{i} t\right)\right), 0 \leq t<\infty$.

Theorem 4.10: Let $\left\{\mathrm{N}_{1, k}(\mathrm{t}), 0 \leq t\right\}, k=1,2, \ldots$, be a sequence of IIDOS processes with mean function $\mathrm{M}_{k}(\cdot), k=1,2, \ldots$, respectively. Suppose $\lim _{k \rightarrow \infty} \mathbb{M}_{k}(t)=\mathbb{M}_{\infty}(t)<\infty, 0 \leq t<\infty$. If $\lim H_{\infty}(t)<\infty$, then the processes converge in distribution to an IIDOS $t \rightarrow \infty$

process with mean function $M_{\infty}(\cdot)$. If $\lim _{t \rightarrow \infty} M_{\infty}(t)=\infty$, then the processes converge in distribution to an NHPP with mean function $M_{\infty}(\cdot)$.

Theorem 4.11: Let $\left\{\mathrm{N}_{2, k}(t), 0 \leq t\right\}, k=1,2, \ldots$, be a sequence of NHPP's with mean functions $\mathrm{M}_{k}(\cdot), k=1,2, \ldots$, respectively. If $\lim _{k \rightarrow \infty} M_{k}(t)=M_{\infty}(t)<\infty, 0 \leq t<\infty$, then the processes converge in distribution to an NHPP with mean function $\mathbb{H}_{\infty}(\cdot)$.

It follows from Theorem 4.9 that the failure times of a DET/EOS process with a finite but large parameter set $\lambda_{\sim}=\left\{\lambda_{1}, \lambda_{2}, \ldots, \lambda_{n}\right\}$ can be approximated by those of a DET/EOS process with an infinite parameter set; this provides some justification for the infinite event models introduced earlier. Theorem 4.10 explains why both IIDOS models and NHPP models exist for bounded mean functions but only the NHPP exists when the mean function is unbounded. All three theorems show that closeness of mean functions implies close distributions of failure times. 
For a given program the deterministic EOS model has certain physical motivation: the individual failure rates are physical quantities in the sense that they can be estimated to any desired degree of accuracy. The IIDOS and NHPP models are attractive because of mathematical tractibility and successful application experience; however, they are somewhat more difficult to motivate and verify in a physical sense. The goal in this section has been to provide justification for these models on the basis of our inability to distinguish among them when a moderately large number of bugs are present and only one debugging replicate can be observed. (The argument is not as airtight as one might wish but does provide some evidence for the conclusion.) Thus we sha11 focus our attention on different reliability growth models as characterized by different mean functions, $M(\cdot)$. (Equivalently, we can consider $\mathrm{m}(\cdot)=\mathbb{H}^{\prime}(\cdot)$, the intensity of the failure process, or $\overline{\mathrm{L}}(\cdot)$, the complementary mean function of the $\Lambda$-process, or $\ell(\cdot)=-\bar{L}^{\prime}(\cdot)$, the intensity (if it exists) of the $\Lambda$-process. Any of the four quantities uniquely determines the other three.)

\section{Gamma Exponential Order Statistic Models}

The Gamma Exponential Order Statistic models ( $\Gamma / E O S)$ are a parametric family of Doubly Stochastic Exponential Order Statistic models; the intensity function of the parameter set $\Lambda$ is

$$
\ell(\lambda)=\gamma \lambda^{\alpha-1} \mathrm{e}^{-\beta \lambda}, 0<\lambda
$$

where $\alpha>-1, \beta>0$, and $\gamma>0$. Recalling that $\overline{\mathrm{L}}(\lambda)=\int_{\lambda}^{\infty} \ell(\mu) \mathrm{d} \mu$ equals the expected number of bugs with rate greater than $\lambda$, we see a dichotomy: if $\alpha>0$, then $\overline{\mathrm{L}}(0)<\infty$; if $-1<\alpha \leq 0$, then $\overline{\mathrm{L}}(0)=\infty$. Thus, for $\alpha>0$, a finite number of events occur and $\Lambda$ may be modeled as an NHPP with intensity $\ell(\cdot)$. If $\alpha>0$ and $\overline{\mathrm{L}}(0)$ is an integer, then $\Lambda$ may instead be an IIDOS process $(\bar{L}(0)$ observations from density $f(\lambda)=\ell(\lambda) / \bar{L}(0))$. When $-1<\alpha \leq 0$ an infinite number of events occur 
and $\Lambda$ will be an NHPP with intensity $\ell(\cdot)$. (Throughout this paper the only stochastic models we consider for $\underset{\sim}{\lambda}$ are NHPP's and IIDOS.)

Now consider the failure process $\{N(t), 0 \geq t\}$, its cumulative mean function $M(\cdot)$, and its intensity $m(\cdot)$ : from Theorems 3.3 and 3.4 ,

$$
\begin{aligned}
& m(t)=\int_{0}^{\infty} \lambda e^{-\lambda t} \ell(\lambda) d \lambda \\
& =\int_{0}^{\infty} \lambda \mathrm{e}^{-\lambda t} \gamma \lambda^{\alpha-1} \mathrm{e}^{-\beta \lambda} \mathrm{d} \lambda \\
& =\gamma \Gamma(\alpha+1)(t+\beta)^{-\alpha-1}, \\
& M(t)=\int_{0}^{t} m(u) d u \\
& =\gamma \Gamma(\alpha+1) \int_{0}^{t}(u+\beta)^{-\alpha-1} d u \\
& =\left\{\begin{array}{l}
\gamma \beta^{-\alpha} \Gamma(\alpha)\left(1-(t / \beta+1)^{-\alpha}\right), \alpha>0 \\
\gamma \log (t / \beta+1), \alpha=0 \\
\gamma \beta^{-\alpha} \Gamma(\alpha+1)(-\alpha)^{-1}\left((t / \beta+1)^{-\alpha}-1\right),-1<\alpha<0 .
\end{array}\right.
\end{aligned}
$$

For $\alpha>0, M(\cdot)$ is proportional to a Pareto cdf; thus if $\Lambda$ is an IIDOS process, then by Theorem $3.3\{N(t), 0 \leq t\}$ is an IIDOS process based on the Pareto distribution, i.e., the Littlewood [16] model. For $-1<\alpha<\infty$, if $\Lambda$ is an NHPP, then by Theorem $3.4\{\mathrm{~N}(\mathrm{t}), 0 \leq t\}$ is an NHPP with mean function $M(\cdot)$ : for $\alpha>0$ we might call it a Pare to NHPP; for $\alpha=0$, we get the Musa-Okumoto [20] Logarithmic NHPP; 
for $-1<\alpha<0$, we get something that could be called a Generalized Power Law NHPP.

The closure of the $\Gamma / E O S$ family consists of the limits obtained as $\alpha \rightarrow-1, \alpha \rightarrow+\infty, \beta \rightarrow 0, \beta \rightarrow+\infty, \gamma \rightarrow 0$, or $\gamma \rightarrow+\infty$. By Theorems 4.10 and 4.11 it suffices to consider limiting behavior of sequences of mean functions. Two possible degenerate limits are $\mathrm{M}_{0}(t) \equiv 0,0 \leq \mathrm{t}<\infty$, and $\mathbb{M}_{\infty}(t)=\infty, 0 \leq t<\infty$, the former corresponding to no failures in $[0, \infty)$ and the latter corresponding to all failures occurring at $t=0$. Nondegenerate limits will have a finite expected number of failures in a finite time interval, for example, $M\left(t_{0}\right)=n_{0}$ for some $0<t_{0}<\infty$ and $0<n_{0}<\infty$. Thus, to find nondegenerate limits, we can consider sequences of $\Gamma /$ EOS mean functions $\left\{\mathrm{I}_{k}(\cdot), k=1,2, \ldots\right\}$ such that $M_{k}\left(t_{0}\right)=n_{0}, k=1,2, \ldots$. The mean functions in Equation (5.3) become

$$
M(t)=\left\{\begin{array}{ll}
n_{0} \frac{1-(t / \beta+1)^{-\alpha}}{1-\left(t_{0} / \beta+1\right)^{-\alpha}}, & \alpha>0 \\
n_{0} \frac{\log (t / \beta+1)}{\log \left(t_{0} / \beta+1\right)}, & \alpha=0 \\
n_{0} \frac{(t / \beta+1)^{-\alpha}-1}{\left(t_{0} / \beta+1\right)^{-\alpha}-1} & -1<\alpha<0
\end{array} .\right.
$$

Without loss of generality let $t_{0}=1$ and consider the following limits: for $0<t<\infty$,

$$
\begin{aligned}
& \lim _{\beta \rightarrow 0} \frac{(t / \beta+1)^{-\alpha}-1}{(1 / \beta+1)^{-\alpha}-1}=t^{-\alpha}, \quad-1<\alpha<0 \\
& \lim _{\beta \rightarrow \infty} \frac{(t / \beta+1)^{-\alpha}-1}{(1 / \beta+1)^{-\alpha}-1}=t \quad, \quad-1<\alpha<0 \\
& \lim _{\beta \rightarrow 0} \frac{\log (t / \beta+1)}{\log (1 / \beta+1)}=1
\end{aligned}
$$




$$
\begin{array}{ll}
\lim _{\beta \rightarrow \infty} \frac{\log (t / \beta+1)}{\log (1 / \beta+1)} & =t \\
\lim _{\beta \rightarrow 0} \frac{1-(t / \beta+1)^{-\alpha}}{1-(1 / \beta+1)^{-\alpha}}=1, & 0<\alpha<\infty \\
\lim _{\beta \rightarrow \infty} \frac{1-(t / \beta+1)^{-\alpha}}{1-(1 / \beta+1)^{-\alpha}}=t, & 0<\alpha<\infty \\
\lim _{\alpha \rightarrow-1} \frac{(t / \beta+1)^{-\alpha}-1}{(1 / \beta+1)^{-\alpha}-1}=t, & 0<\beta<\infty \\
\lim _{\alpha \rightarrow 0} \frac{(t / \beta+1)^{-\alpha}-1}{(1 / \beta+1)^{-\alpha}-1}=\frac{\log (t / \beta+1)}{\log (1 / \beta+1)}, 0<\beta<\infty \\
\lim _{\alpha \vee 0} \frac{1-(t / \beta+1)^{-\alpha}}{1-(1 / \beta+1)^{-\alpha}} \\
\lim _{\alpha \rightarrow \infty} \frac{1-(t / \beta+1)^{-\alpha}}{1-(1 / \beta+1)^{-\alpha}}=\frac{\log (t / \beta+1)}{\log (1 / \beta+1)}, & 0<\beta<\infty \\
& =1,0<\beta<\infty .
\end{array}
$$

(The above limits are verified by using l'Hopital's Rule in various forms.) The NHPP with mean function $M(t)=n_{0} t^{-\alpha},-1<\alpha<0$, is the Power Law NHPP studied by Crow [6] and Duane [7]. The NHPP with mean function $M(t)=n_{0} t$ is the homogeneous Poisson process (HPP). The NHPP or IIDOS process with mean function $M(t)=n_{0} \cdot 1$ is a degenerate case with all (finite number) failures occurring at $t=0$. In terms of models, the above limits have the following interpretations:

$$
\begin{aligned}
& \text { Generalized Power Law NHPP }(\alpha, \beta) \\
& \quad \Rightarrow \text { Power Law NHPP }(\alpha) \text {, as } \beta \rightarrow 0 \\
& \text { Generalized Power Law NHPP }(\alpha, \beta) \\
& \quad \Rightarrow \text { HPP, as } \beta \rightarrow \infty \\
& \text { Musa-Okumoto Logarithmic NHPP }(\beta) \\
& \quad \Rightarrow \text { Degenerate }(0) \text {, as } \beta \rightarrow 0
\end{aligned}
$$


Musa-okumoto Logarithmic NHPP ( $\beta$ )

$\Rightarrow \mathrm{HPP}$, as $\beta \rightarrow \infty$

Littlewood's Pareto IIDOS model $(\alpha, \beta)$

$\Rightarrow$ Degenerate IIDos (0) model, as $\beta \rightarrow 0$

Pareto NHPP $(\alpha, \beta)$

$\Rightarrow$ Degenerate NHPP (0), as $\beta \rightarrow 0$

Littlewood's Pareto IIDOS model $(\alpha, \beta)$

$\Rightarrow \mathrm{HPP}$, as $\beta \rightarrow \infty$

Pareto NHPP $(\alpha, \beta)$

$\Rightarrow \mathrm{HPP}$, as $\beta \rightarrow \infty$

Generalized Power Law NHPP $(\alpha, \beta)$

$\Rightarrow \mathrm{HPP}$, as $\alpha \searrow-1$

Generalized Power Law NHPP $(\alpha, \beta)$

$\Rightarrow M-0$ Logarithmic NHPP $(\beta)$, as $\alpha>0$

$\Rightarrow M-0$ Logarithmic $\operatorname{NHPP}(\beta)$, as $\alpha \vee 0$

Pareto NHPP $(\alpha, \beta)$

$\Rightarrow M-0$ Logarithmic NHPP $(\beta)$, as $\alpha \searrow 0$

Littlewood's Pareto IIDOS model $(\alpha, \beta)$

$\Rightarrow$ Degenerate IIDOS mode1 (0), as $\alpha \rightarrow \infty$

Pareto NHPP $(\alpha, \beta)$

$\Rightarrow$ Degenerate NHPP (0), as $\alpha \rightarrow \infty$.

The above results follow from Theorems 4.10 and 4.11 and the appropriate limits (Equations (5.5) through (5.14)). The relationship of the above models is summarized in Figure 5.1. Note that the Power Law NHPP is an NHPP/EOS process whose $\Lambda$-process has intensity

$$
\ell(\lambda)=\gamma \lambda^{\alpha-1}, \quad 0<\lambda
$$




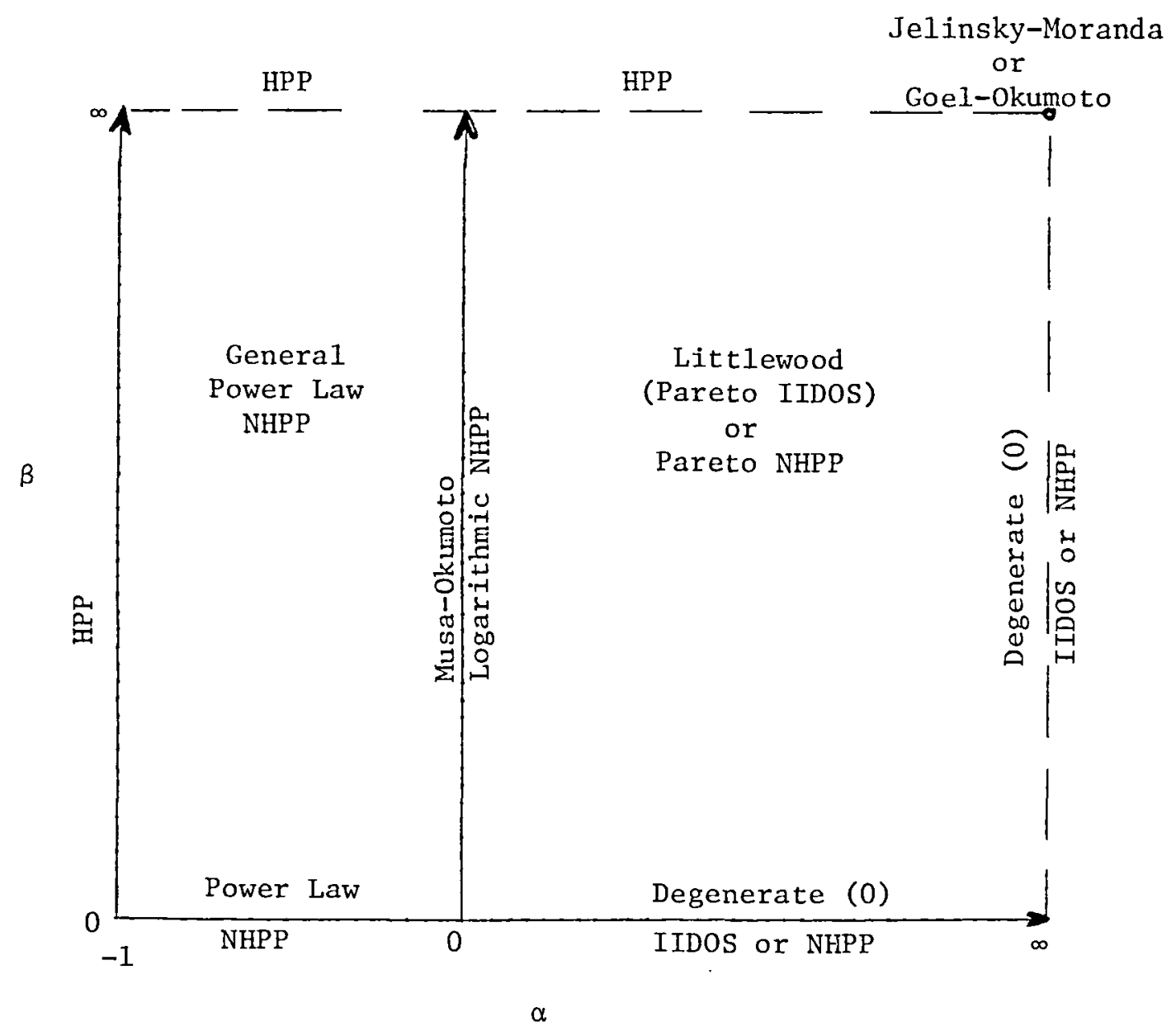

Figure 5.1 Gamma EOS models and 1imits: a parametric family that includes several we11-known models. The parameters $\alpha$ and $\beta$ take values in $[-1, \infty] \times[0, \infty]$. If $(\alpha, \beta) \rightarrow$ $(\infty, \infty)$ but $\alpha / \beta \rightarrow \eta$, the parameterization is $n$ instead of $(\alpha, \beta)$. The third parameter is always $\gamma, 0<\gamma$. 
for $-1<\alpha<0$. Note, however, that an HPP cannot be represented as a DS/EOS process because it shows no growth. The Degenerate (0) NHPP and IIDOS processes can be considered as EOS models if and only if $\lambda=\infty$ is allowed in the definition of EOS models.

Figure 5.1 shows that we have a nearly complete picture of $\Gamma /$ EOS models and their limits, which are normalized so that $M\left(t_{0}\right)=n_{0}$ for some $t_{0}$ and $n_{0}$. The only remaining case consists of limits when $\alpha \rightarrow \infty$ and $\beta \rightarrow \infty$ simultaneously. Suppose that $\beta=\alpha / n, 0<n<\infty$; then we have

$$
\lim _{\alpha \rightarrow \infty} \frac{1-(n t / \alpha+1)^{-\alpha}}{1-(n / \alpha+1)^{-\alpha}}=\frac{1-e^{-n t}}{1-e^{-n}},
$$

which, analogous to the above limits, is interpreted as

$$
\begin{aligned}
& \text { Littlewood's Pareto IIDOS }(\alpha, \alpha / \eta) \text { model } \\
& \quad \Rightarrow \text { Jelinsky-Moranda }(\eta) \text { model, as } \alpha \rightarrow \infty \\
& \text { Pareto NHPP }(\alpha, \alpha / \eta) \\
& \quad \Rightarrow \text { Goel-okumuto }(\eta) \text { model, as } \alpha \rightarrow \infty .
\end{aligned}
$$

Thus the parametric family of $\Gamma / E O S$ models and their limits is very rich, including some very well-known models as subcases. It might be fruitful to think of these models as special cases of the general three-parameter family, where $\alpha$ is a "shape" parameter, $-1 \leq u \leq \infty, \beta$ is a "scale" parameter, $0 \leq \beta \leq \infty$, and $\gamma$ is a "magnitude" parameter, $0<\gamma$.

Figures 5.2 through 5.11 show the mean functions for various $(\alpha, \beta, \gamma)$ in the parametric family of Figure 5.1. Al1 mean functions are normalized so that $\mathrm{M}(100)=100$. The purpose is to convey the extent and the richness of this family as well as to provide a visual comparison between the shapes of the mean functions for different subfamilies of the entire family. 


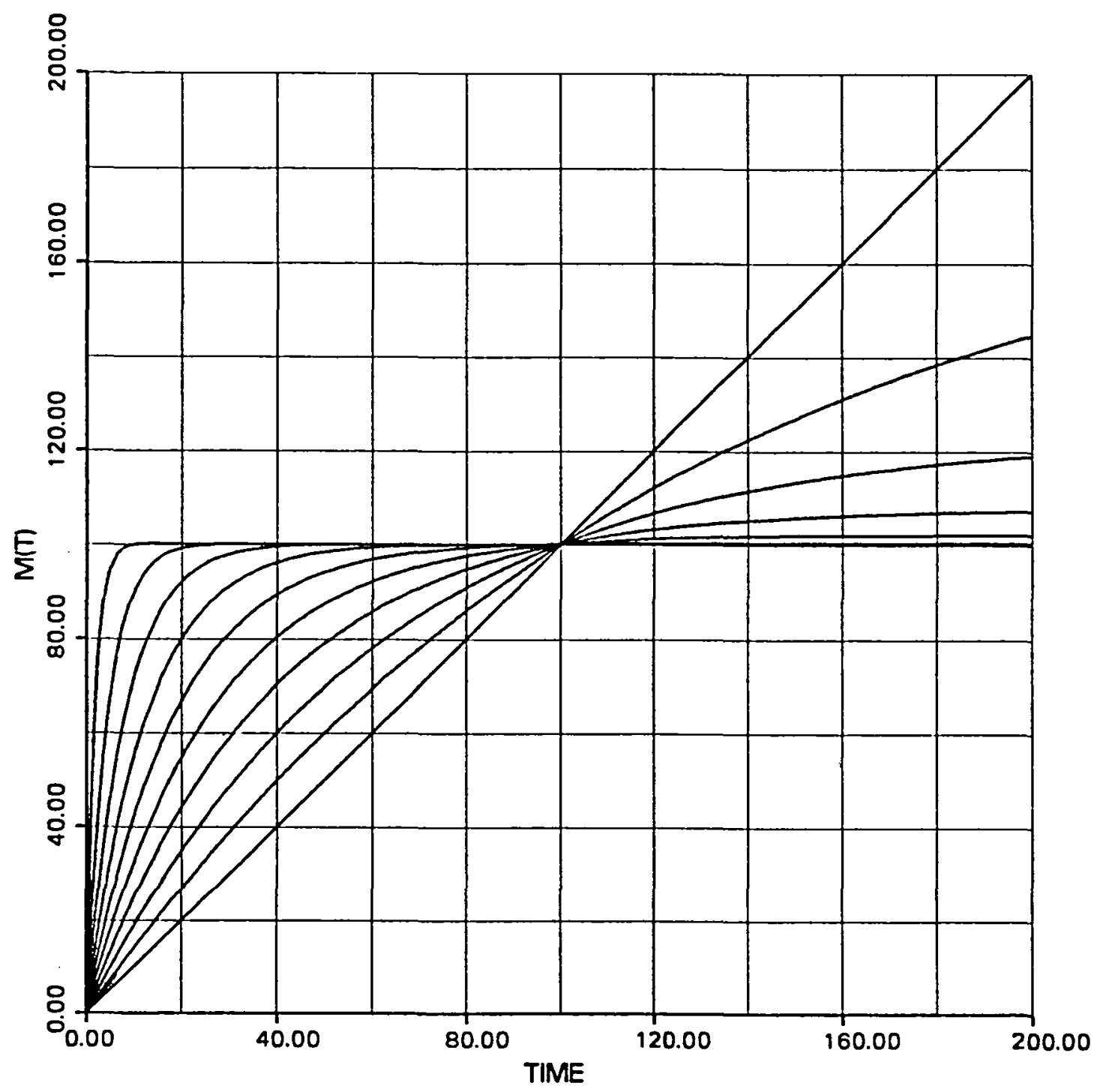

Figure 5.2 $\Gamma /$ EOS Mean Functions, Exponential

Case $(\alpha / \beta=n, \alpha=\infty, \beta=\infty)$;

$\eta=0, .00808, .0167, .0265$,

$.0385, .0550, .804, .126, .230$,

.600 




Figure 5.3 $\Gamma /$ EOS Mean Functions, Pareto Case; $\alpha=16, \beta=\infty, 2060 ., 977 ., 604$. , 407., 281., 190., 119., 64.6, 24.3 


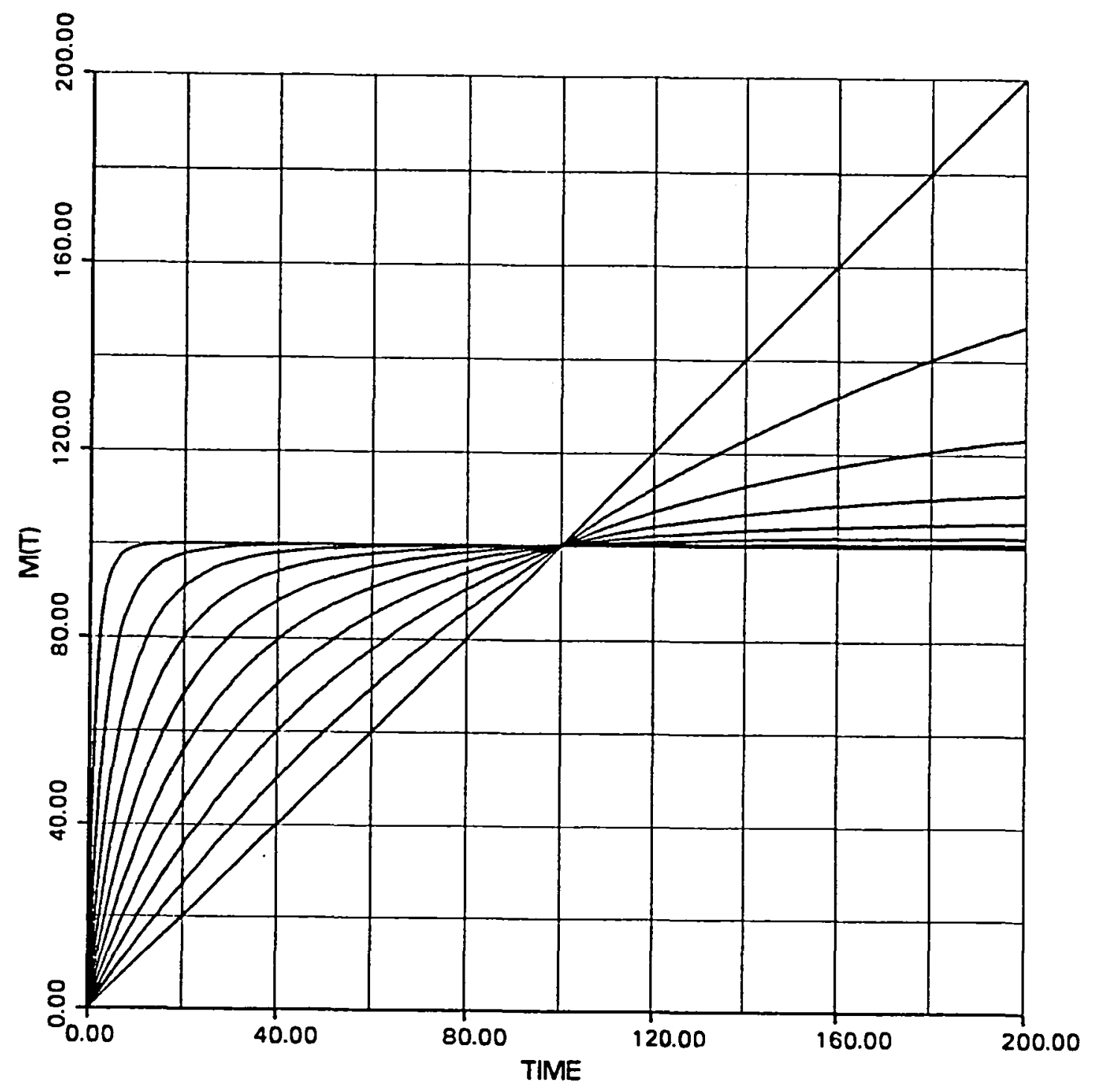

Figure 5.4 Г/EOS Mean Functions, Pareto Case;

$$
\begin{gathered}
\alpha=4, \beta=\infty, 573 ., 258 ., 152 ., 97.3, \\
64.0,41.3,24.9,12.9,4.49
\end{gathered}
$$






Figure 5.5 T/EOS Mean Functions, Pareto Case; $\alpha=1, \beta=\infty, 203 ., 80.0,40.8$, $22.5,12.5,6.67,3.21,1.25, .278$ 


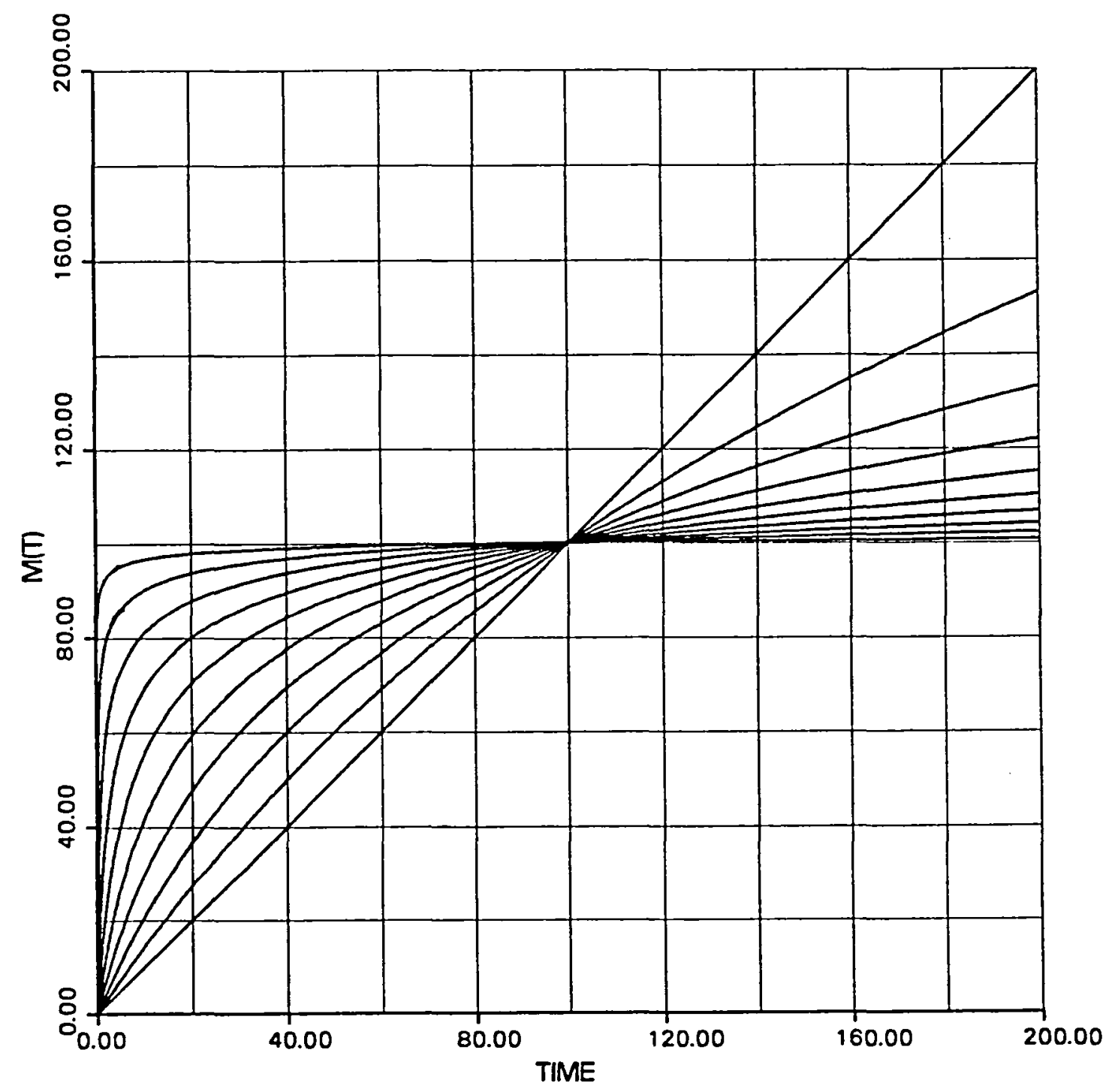

Figure 5.6 T/EOS Mean Functions, Pareto Case;

$$
\begin{gathered}
\alpha=.25, \beta=\infty, 111 ., 36.9,15.2, \\
6.29,2.37, .734, .157, .0169, .00034
\end{gathered}
$$




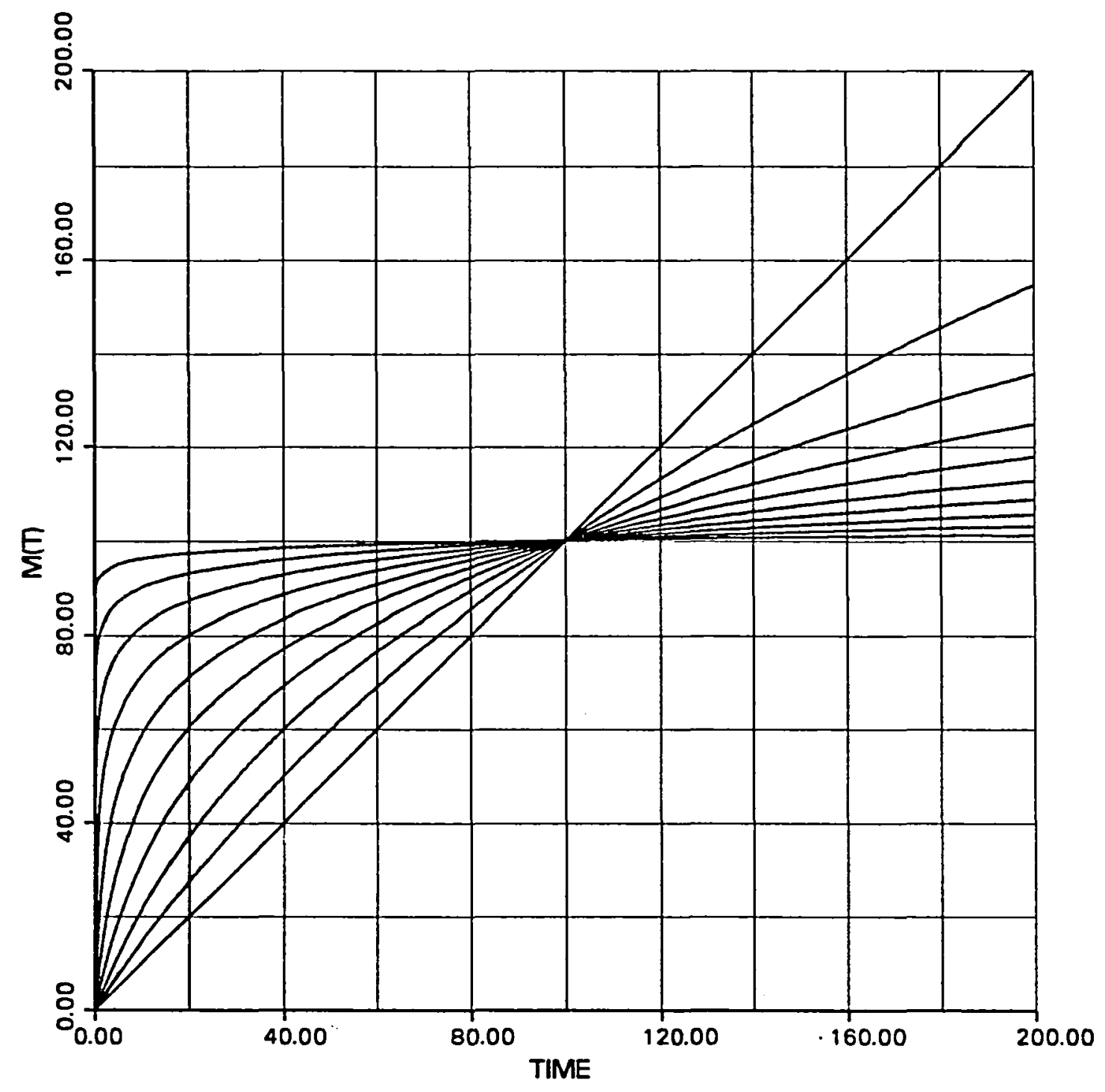

Figure 5.7 T/EOS Mean Functions, Logarithmic Case;

$\alpha=0, \beta=\infty, 80.4,23.3,7.64$,

$2.17, .412, .0322, .000322$,

$.100 \times 10^{-7}, .960 \times 10^{-24}$ 




Figure 5.8 I/EOS Mean Functions, Generalized Power Case; $\alpha=-1 / 32, \quad \beta=\infty, 76.6,21.6,6.77,1.76$, $.272, .0123, .190 \times 10^{-4}, .345 \times 10^{-14}, 0$ 


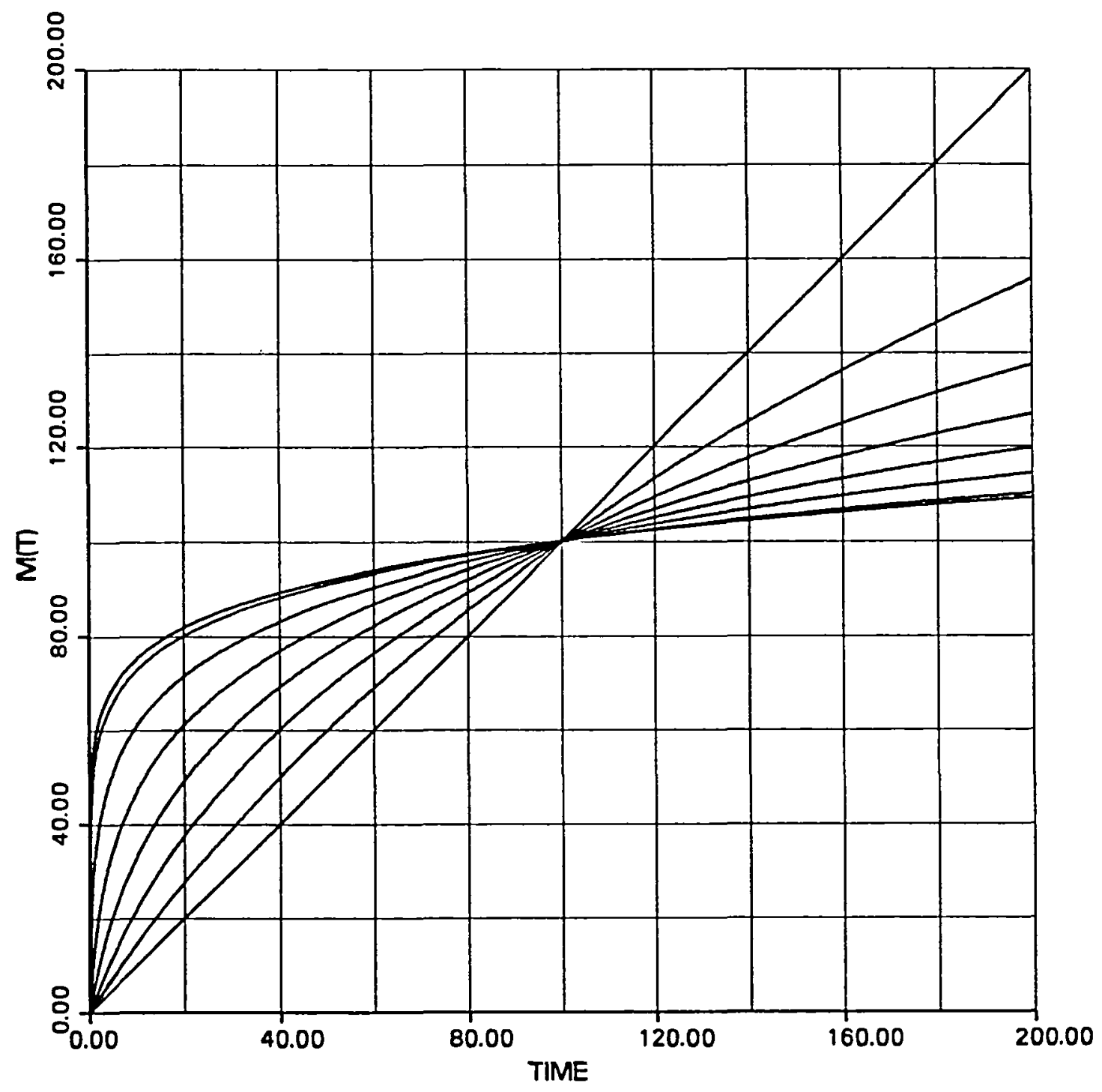

Figure 5.9 Г/EOS Mean Functions, Generalized Power Case; $\alpha=-1 / 8, \quad \beta=\infty, 65.4,16.7,4.34, .732$, $.0308, .388 \times 10^{-6}, 0$ 




Figure 5.10 T/EOS Mean Functions, Generalized Power Case; $\alpha=-1 / 2, \quad \beta=\infty, 22.0, .835,0$ 


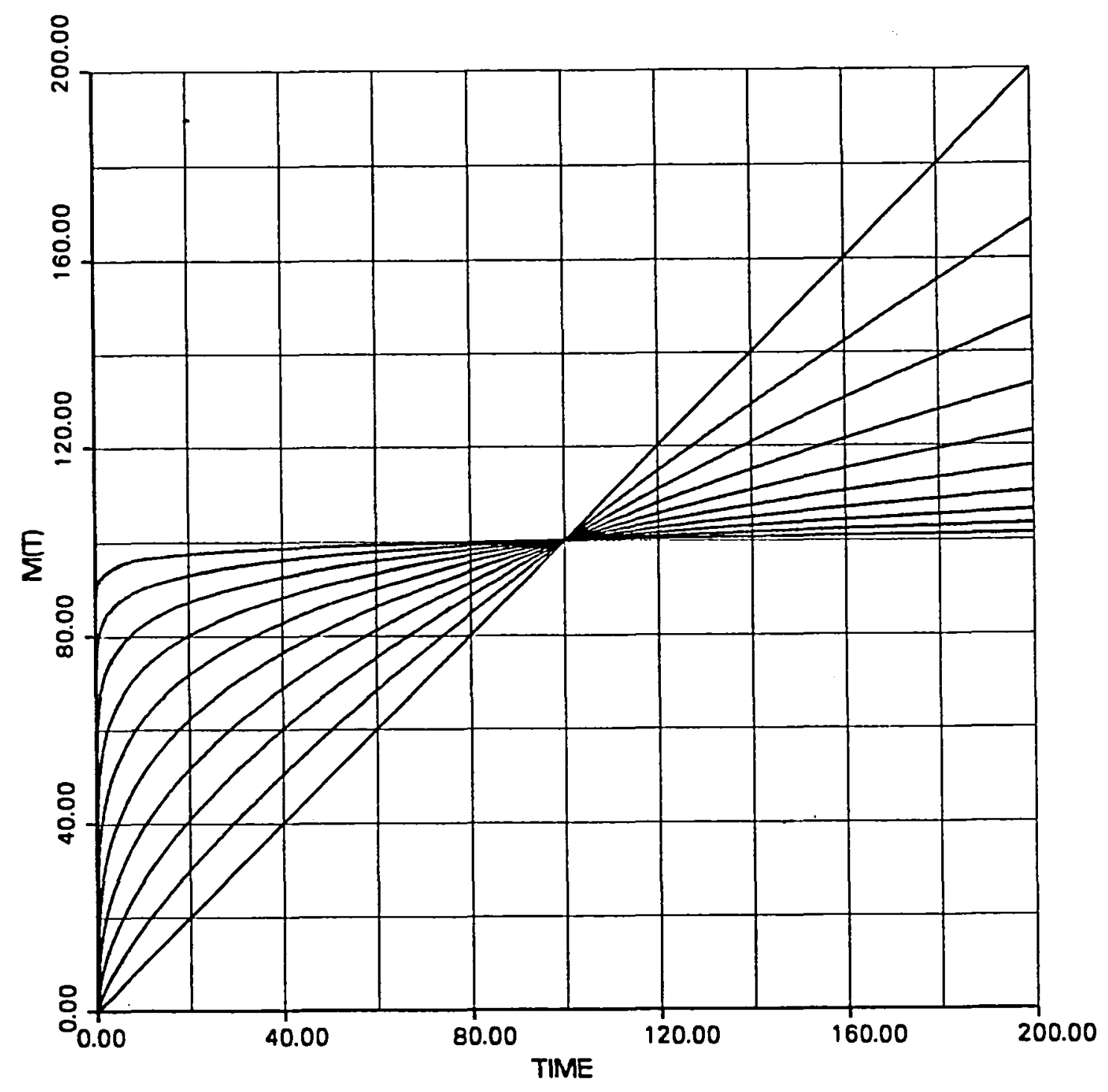

Figure 5.11 T/EOS Mean Functions, Power Case;

$\beta=0, \alpha=-1.000,-.749,-.557$,

$-.410,-.296,-.208,-.139,-.086$,

$-.046,-.017$ 
The above limit properties, i.e., the continuity in $\alpha$ and $\beta$ of the models in the above parametric family, have certain ramifications. In particular, if $\left(\alpha_{1}, \beta_{1}, \gamma_{1}\right)$ and $\left(\alpha_{2}, \beta_{2}, \gamma_{2}\right)$ are close to each other the respective models with these parameters will produce failure data that appear to be similar. For example, if the true model is Littlewood's Pareto IIDOS model with very large $\alpha$ and $\beta$ and $\alpha / \beta=n$, because of statistical variation in the data, the Jelinsky-Moranda ( $n$ ) model might often provide a better fit to the data; the converse is also possible. Another possible case of mistaken identity could involve Littlewood's IIDOS model with shape parameter $\alpha$ close to zero and the Musa-Okumoto logarithmic NHPP with the same value of $\beta$; this case is of added interest because it involves a finite event model and an infinite event model. Typical failure data are over a finite interval; if $\alpha$ is close enough to zero it is quite possible that the infinite event $M$ - 0 model would fit data from the finite event Littlewood model better than the Littlewood model itself. The continuity of the models around $\alpha=0$ means that there is no sharp demarcation between infinite and finite event models for failure data on a finite interval. This continuity and the common $(\alpha, \beta, \gamma)$-parameterization of the models in Figure 5.1 suggests that it is appropriate to consider them as one model: Perhaps we should call it the J-M-G-O-L-M-O-D-C Model (Jelinsky-Moranda Goel-Okumoto-Littlewood-Musa-Okumoto-Duane-Crow Mode1).

\section{Complete Monotonicity Property}

The DET/EOS, the IIDOS/EOS, and the NHPP/EOS processes are a11 characterized by their cumulative mean functions or, equivalently, by their intensity functions; i.e., the mean function $M(\cdot)$, or intensity $\mathrm{m}(\cdot)$, and the family (deterministic, IIDOS, or NHPP) to which $\Lambda$ belongs completely determine the distribution of the failure process $\{N(t), 0 \leq t\}$. For NHPP/EOS models, the class of possible intensities will be proven to be exactly the class of positive completely monotone functions such that $\lim _{t \rightarrow \infty} m(t)=0$ and $M(t)=\int_{0}^{t} m(u) d u<\infty, 0 \leq t<\infty$. 
Intensities for DET/EOS models and IIDOS/EOS models are "almost dense" subsets of this class.

First, the family of admissible $\overline{\mathrm{L}}(\cdot)^{\prime}$ 's must be characterized. Recall that $\overline{\mathrm{L}}(\lambda)$ equals the expected number of bugs wi th rates greater than or equal to $\lambda ; \overline{\mathrm{L}}(\cdot)$ must be a nonincreasing function on $[0, \infty]$. The following three theorems will characterize admissible $\bar{L}(\cdot)^{\prime}$ s:

Theorem 6.1: Let $\left\{\lambda_{1}, \lambda_{2}, \ldots\right\}$ be a deterministic process wi th complementary mean function $\overline{\mathrm{L}}(\cdot)$; then $\sum_{i=1}^{\infty} \lambda_{i}<\infty$ if and only if $\lim _{\lambda \rightarrow \infty} \bar{L}(\lambda)=0$ and $\int_{0}^{\mu} \bar{L}(\lambda) \mathrm{d} \lambda<\infty, 0 \leq \mu<\infty$.

Theorem 6.2: Let $\left\{\Lambda_{1}, \Lambda_{2}, \ldots, \Lambda_{n}\right\}$ be an IIDOs process with complementary mean function $\overline{\mathrm{L}}(\cdot)$; then $\mathrm{P}\left(\sum_{i=1} \Lambda_{i}<\infty\right)=1$ if and only if $\lim _{\lambda \rightarrow \infty} \overline{\mathrm{L}}(\lambda)=0$.

Theorem 6.3: Let $\left\{\Lambda_{1}, \Lambda_{2}, \ldots\right\}$ be an NHPP with complementary mean function $\tilde{\mathrm{L}}(\cdot)$; then $\mathrm{P}\left(\sum_{i=1}^{\infty} \Lambda_{i}<\infty\right)=1$ if and only if $\lim _{\lambda \rightarrow \infty} \overline{\mathrm{L}}(\lambda)=0$ and $\int_{0}^{\mu} \overline{\mathrm{L}}(\lambda) \mathrm{d} \lambda<\infty, 0 \leq \mu<\infty$.

A proper EOS process must have $\sum \lambda_{i}<\infty$; if the $\lambda^{\prime}$ 's are a realization of a stochastic process $\left\{\Lambda_{1}, \Lambda_{2}, \ldots\right\}$ then $\Sigma \Lambda_{i}$ must be finite with probability one. So Theorem 6.1 through 6.3 lead to the following definition:

Definition 0.1: A complementary cumulative mean function $\overline{\mathrm{L}}(\cdot)$ of a $\Lambda$-process is admissible if $\lim _{\lambda \rightarrow 0} \bar{L}(\lambda)=0$, and $\int_{0}^{\mu} \bar{L}(\lambda) d \lambda<\infty, 0<\mu<\infty$ 
If the process is deterministic $\overline{\mathrm{L}}(\cdot)$ must be integer-valued. For an IIDOS process $\overrightarrow{\mathrm{L}}(0)$ must be integer-valued. (We use the convention that "admissible" refers to NHPP; restriction to deterministic and IIDOS models is discussed later.)

Theorem 6.4: If $\overline{\mathrm{L}}(\cdot)$ is admissible then

$$
m(t)=-\int_{0}^{\infty} \lambda e^{-\lambda t} d \bar{L}(\lambda)<\infty, 0<t<\infty
$$

Furthermore, $\overline{\mathrm{L}}(\cdot)$ is admissible if and only if

$$
M(t)=-\int_{0}^{\infty}\left(1-e^{-\lambda t}\right) d \bar{L}(\lambda)<\infty, 0 \leq t<\infty,
$$

and $\lim _{t \rightarrow 0} M(t)=0$

Thus when $\overline{\mathrm{L}}(\cdot)$ is admissible the intensity and mean function are well defined and uniquely determine the distribution of the DET/EOS, IIDOS, or NHPP model. We wish to characterize the family of admissible intensities or the family of admissible mean functions.

The definition of complete monitonicity and an important theorem relating completely monotone functions to Laplace transforms are given by Feller [9, p. 439]:

Definition 6.2: A function $\phi(\cdot)$ on $[0, \infty]$ is completely monotone if it possesses derivatives $\phi^{(n)}(\cdot)$ of all orders and

$$
(-1)^{n} \phi^{(n)}(t) \geq 0,0<t<\infty .
$$

Theorem 6.5: The function $\phi(\cdot)$ on $[0, \infty]$ is completely monotone iff it is of the form

$$
\phi(t)=\int_{0}^{\infty} e^{-\lambda t} d F(\lambda), 0<t<\infty,
$$


where $F$ is a measure on $[0, \infty]$ which may be finite or infinite. Comparison of Equations (6.1) and (6.4) suggest that we define

$$
F(\mu)=-\int_{0}^{\mu} \lambda \mathrm{d} \overline{\mathrm{L}}(\lambda), \quad 0 \leq \mu<\infty,
$$

which is the cmulative function for a measure on $[0, \infty)$; integrability of (6.5) follows from admissibility of $\overline{\mathrm{L}}(\cdot)$ and the argument for integration by parts in the proof of Theorem 6.4. Thus any admissible intensity function $\mathrm{m}(\cdot)$ has the form of Equation (6.4) and must be completely monotone. Conversely consider any completely monotone function of the form (6.4) which is integrable in intervals around 0 : let

$$
\begin{aligned}
\infty>\Phi(t) & =\int_{0}^{t} \phi(u) d u \quad, 0 \leq t<\infty \\
& =\int_{0}^{t} \int_{0}^{\infty} e^{-\lambda u} d F(\lambda) d u \\
& =\int_{0}^{t} F(0) d u+\int_{0}^{t} \int_{0}^{+} e^{-\lambda u} d F(\lambda) d u \\
& =t F(0)+\int_{0}^{\infty} \lambda^{-1}\left(1-e^{-\lambda t}\right) d F(\lambda)
\end{aligned}
$$

Letting $\lambda^{-1} \mathrm{dF}(\lambda)=-\mathrm{d} \overline{\mathrm{L}}(\lambda)$, we recognize Equation (6.2) when there is no atom at 0 , i.e., $F(0)=0$. Under this restriction, Theorem 6.4 guaranties admissibility of $\overline{\mathrm{L}}(\cdot)$ and consequently $\Phi(\cdot)$ is an admissible mean function. It is seen from Equation (6.4) and the monotone convergence theorem that $F(0)=0$ is equivalent to $\lim \phi(t)=0$. Summarizing gives the following theorem: $t \rightarrow \infty$

Theorem 6.6: A positive function $\mathrm{m}(\cdot)$ is an admissible intensity (for an NHPP/EOS process) if and only if 


$$
\begin{aligned}
& \text { (i) } m(\cdot) \text { is completely monotone on }[0, \infty] \\
& \text { (ii) } \lim _{t \rightarrow \infty} m(t)=0 \text {, and } \\
& \text { (iii) } \int_{0}^{t} m(u) d u<\infty, 0 \leq t<\infty \text {. }
\end{aligned}
$$

Note that condition (iii) in the above theorem is automatically satisfied if $m(0)<\infty$ and the counterexample $m(t)=1 / t$ shows it to be necessary. Theorem 6.6 can be restated for the mean function.

Theorem 6.7: A positive function $M(\cdot)$ is an admissible mean function (for an NHPP/EOS process) if and only if

$$
\begin{aligned}
& \text { (i) } M(0)=0 \\
& \text { (ii) } \lim _{t \rightarrow \infty} M^{\prime}(t)=0 \text {, and } \\
& \text { (iii) }(-1)^{n} \mathbb{H}^{(n)}(t) \leq 0,0<t<\infty, n \geq 1 .
\end{aligned}
$$

Now consider the families of admissible mean functions and intensities for the failure-time processes of DET/EOS and IIDOS/EOS models. These are subsets of the admissible functions of Theorems 6.6 and 6.7 in a way very similar to that in which the integers are a subset of the real numbers. Properties of these subsets are given in the following theorems:

Theorem 6.8: If $M(\cdot)$ is an admissible mean function for an NHPP/EOS process with $M(\infty)<\infty$, then $M_{1}(t)=([M(\infty)+1 / 2] / M(\infty)) M(t)$, $0 \leq t<\infty$ is an admissible mean function for an IIDOS/EOS process. Thus for every bounded NHPP/EOS - admissible mean function $M(\cdot)$ there exists an IIDOS/EOS - admissible mean function $M_{1}(\cdot)$ such that $\sup \left|M(t)-\mathrm{II}_{1}(t)\right| \leq 1 / 2$. Furthermore, $m(t)=M^{\prime}(t)$ and $m_{1}(t)=M_{1}^{\prime}(t)$ satisfy $\left|m(t)-m_{1}(t)\right| \leq(2 e t)^{-1}, 0 \leq t<\infty$. 
Theorem 6.9: If $M(\cdot)$ is an admissible mean function for an NHPP/EOS process whose $\Lambda$-process has complementary cumulative mean function $\overline{\mathrm{L}}(\cdot)$, then the DET/EOS process with $\lambda=\left\{\lambda_{i}=\bar{L}^{-1}(i-1 / 2), i=1,2, \ldots\right\}$ has cumulative mean function $M_{0}(\cdot)$ which satisfies $\sup _{t}\left|M(t)-M_{0}(t)\right|<1$. Furthermore, $m(t)=M^{\prime}(t)$ and $m_{0}(t)=M_{0}^{\prime}(t)$ satisfy $\left|m(t)-m_{0}(t)\right| \leq 2 /(e t)$, $0 \leq \mathrm{t}<\infty$

These two theorems provide a sense in which the admissible functions for DET/EOS and IIDOS/EOS models are "almost dense" within the set of admissible functions for NHPP/EOS processes characterized in Theorems 6.6 and 6.7 . When the expected number of bugs is moderately large (50 or more, say), the above $\pm 1 / 2$ and \pm 1 terms seem negligible, obviating the need to make the restriction to the above subsets, especially when considering mean functions; thus we could use the characterizations in Theorems 6.6 or 6.7 for all three types of models (DET/EOS, IIDOS/EOS, or NHPP/EOS).

If the failure rates of an EOS model are all less than or equal to 1 , i.e., $0<\lambda \leq 1$, then complete monotonicity appears again in a different context. The result follows from material found in Feller [9, pp. 224-226]:

Definition 6.3: A sequence $\left\{c_{k}, k=0,1,2, \ldots\right\}$ is completely monotone if its differences alternate in sign, i.e.,

$$
(-1)^{r} \Delta^{r} c_{k} \geq 0, r \geq 0, k \geq 0
$$

Theorem 6.10: The moments $c_{k}$ of a probability distribution on $[0,1]$ form a completely monotone sequence with $c_{0}=1$. Conversely, an arbitrary completely monotone sequence with $c_{0}=1$ coincides 
with the moment sequence of a unique probability distribution.

From the Taylor series expansion for $e^{-\lambda t}$ we get

$$
\begin{aligned}
& m(t)=\sum_{i=0}^{\infty}\left(-\int_{0}^{1} \lambda^{i} \lambda d \bar{L}(\lambda)\right)(-t)^{i} / i !, \\
& M(t)=\sum_{i=0}^{\infty}\left(-\int_{0}^{1} \lambda^{i} \lambda d \bar{L}(\lambda)\right)(-1)^{i} t^{i+1} /(i+1) !
\end{aligned}
$$

Letting

$$
\begin{aligned}
\mu_{i} & =-\int_{0}^{1} \lambda^{i} \lambda \mathrm{d} \overline{\mathrm{L}}(\lambda) / c, i \geq 0, \\
c & =-\int_{0}^{1} \lambda \mathrm{d} \overline{\mathrm{L}}(\lambda),
\end{aligned}
$$

we get

$$
\begin{aligned}
& m(t)=c \sum_{i=0}^{\infty}(-1)^{i} \frac{\mu_{i} t^{i}}{i !} \\
& M(t)=c \sum_{i=0}^{\infty}(-1)^{i} \frac{\mu_{i} t^{i+1}}{(i+1) !}
\end{aligned}
$$

Admissibility of $\overline{\mathrm{L}}(\cdot)$ implies that $c<\infty ; \mu_{i}$ is the ith moment of a distribution on $[0,1]$ with $\mathrm{dF}(\lambda)=-\lambda \mathrm{d} \overline{\mathrm{L}}(\lambda) / \mathrm{c}$. By Theorem 6.10 $\left\{\mu_{i}, i=0,1, \ldots\right\}$ is a completely monotone sequence. Thus, the Taylor series expansions in Equations (6.8) and (6.9) must have completely monotone coefficients whenever $\overline{\mathrm{L}}\left(1^{+}\right)=0$. Conversely, by Theorem 6.10 , if $\left\{v_{i}\right\}$ is a completely monotone sequence it must be a moment sequence of a distribution $F(\cdot)$ on $[0,1]$. If $F(0)=0$, then, as before, there exists an admissible $\overline{\mathrm{L}}(\cdot)$ such that 
$\mathrm{dF}(\lambda)=-\operatorname{a\lambda } \mathrm{d} \overline{\mathrm{L}}(\lambda), 0 \leq \lambda \leq 1$, and $\psi(t)=\frac{1}{\mathrm{a}} \sum_{i=0}^{\infty}(-1)^{i} \nu_{i} \mathrm{t}^{i} / \mathbf{i}$ : and $\Psi(t)=\frac{1}{a} \sum_{i=0}^{\infty}(-1)^{i} \nu_{i} t^{i+1} /(i+1) ! \quad$ will be the intensity and mean function, respectively, of an EOS process. As before, $\lim _{t \rightarrow \infty} \psi(t)=0$ will be necessary and sufficient to guarantee $F(0)=0$ and consequently the existence of an admissible $\overline{\mathrm{L}}(\cdot)$. In summary we have the following characterization:

Theorem 6.11: The intensity function and mean function of any EOS process with $\overline{\mathrm{L}}\left(1^{+}\right)=0$ have the form

$$
\begin{aligned}
& m(t)=\sum_{i=0}^{\infty}(-1)^{i} v_{i} t^{i} / i ! \\
& M(t)=\sum_{i=0}^{\infty}(-1)^{i} v_{i} t^{i+1} /(i+1) !,
\end{aligned}
$$

respectively, where $\left\{v_{i}\right\}$ is a completely monotone sequence such that

$$
\lim _{t \rightarrow \infty} \sum_{i=0}^{\infty}(-1)^{i} v_{i} t^{i} / i:=0
$$

Conversely, for any completely monotone sequence $\left\{\nu_{i}\right\}$ satisfying (6.15), the functions in (6.13) and (6.14) will be the intensity and mean function, respectively, of an EOS model whose rates are all less than 1 .

By rescaling the time-axes (e.g., measuring time in nanoseconds instead of seconds), it is possible to satisfy the condition $\overline{\mathrm{L}}\left(1^{+}\right)=0$ with a high degree of confidence. (However, if time is discrete and indicates the indices of repeated trials, then we may not be able to rescale and should perhaps consider a Geometric Order Statistic mode1; see Appendix.) 


\section{Additional EOS Models and Properties}

The general framework and description of EOS models presented above seems quite fruitful. It leads to new models, new perspectives on familiar models, and other insights. A miscellanea of such results is presented in this section.

NHPP and IIDOS models. From Theorems 3.3 and 3.4 we know that IIDOS/EOS models and NHPP/EOS models are in fact IIDOS models and NHPP models, respectively. The converse is not true; not all IIDOS models can arise as IIDOS/EOS models, nor can all NHPP models arise as NHPP/EOS models. They must have differentiable cdf's or mean functions, respectively, which satisfy the conditions of Theorem 6.7, or equivalently, densities or intensities which satisfy the conditions of Theorem 6.6. This allows us to consider IIDOS models and NHPP models directly without considering the EOS structure. For example, let $\{N(t), 0 \leq t\}$ be an NHPP with intensity

$\mathrm{m}_{1}(t)=a(b+\log (t+c+1))^{-d}$ with $0<a, b, c, d ; m_{1}(\cdot)$ satisfies Theorem 6.6, and therefore $\{\mathrm{N}(\mathrm{t}), 0 \leq t\}$ may arise from an EOS mode1. On the other hand, suppose $m_{2}(t)=1 /\left(x^{2}+2 x+2\right) ;$ this appears to be a plausible intensity, however the fourth derivative is negative for small $t$, violating complete monotonicity. Thus if we believe the EOS modeling paradigm we would not consider an NHPP with intensity $m_{2}(\cdot)$ as a possible model for the failure time process. Feller [9, p. 441] presents some properties which are useful for identification of completely monotone functions.

Weibu11 IIDOS models. The Weibull distribution has cdf

$$
F(x)=1-\exp \left(-(x / \beta)^{\alpha}\right), x \geq 0,
$$

and density

$$
f(x)=\alpha \beta^{-\alpha} x^{\alpha-1} \exp \left(-(x / \beta)^{\alpha}\right), x \geq 0,
$$


where $\alpha>0$ and $\beta>0$. The density $f(\cdot)$ satisfies Theorem 6.6 (or, equivalently, the $\operatorname{cdf} F(\cdot)$ satisfies Theorem 6.7) if and on $1 \mathrm{y}$ if $0<\alpha \leq 1$, independently of the value of $\beta$. Thus, there exist IIDOS/EOS models whose failure time processes are Weibul1 IIDOS with shape parameter $0<\alpha<1$. The mean function for the underlying $\Lambda$-process satisfies

$$
n \alpha \beta^{-\alpha} t^{\alpha-1} \exp \left(-(t / \beta)^{\alpha}\right)=\int_{0}^{\infty} \lambda e^{-\lambda t} d L(\lambda)
$$

Solving (7.3) for $L(\cdot)$ is equivalent to inverting a Laplace transform; the case $\alpha=1 / 2$ can be found in tables of Laplace transforms:

$$
s^{-1 / 2} \exp \left(-k s^{1 / 2}\right)=\int_{0}^{\infty} e^{-\lambda s}(\pi \lambda)^{-1 / 2} \exp \left(-k^{2} / 4 \lambda\right) d \lambda
$$

which gives

$$
\lambda \mathrm{dL}(\lambda)=\mathrm{n} 1 / 2 \beta^{-1 / 2}(\pi \lambda)^{-1 / 2} \exp (-\beta / 4 \lambda)
$$

Thus the $\Lambda$-process is an IIDOS process from a distribution with density

$$
g(\lambda)=(4 \pi \beta)^{-1 / 2} \lambda^{-3 / 2} \exp (-\beta / 4 \lambda)
$$

If $\alpha \neq 1 / 2$, there appears to be no closed-form solution for $d L(:)$ in Equation (7.3); however, this does not prevent use of the Weibull IIDOs model for $\{N(t), 0 \leq t\}$. Thus we can identify possible models for the failure time process of EOS models without detailed knowledge of a model of the underlying $\Lambda$-process. Musa and okumoto [20] introduce an NHPP with intensity proportional to $f(\cdot)$ of Equation (7.2). By the above analysis this is an acceptable model for the failure times of an EOS process, whenever $0<\alpha \leq 1$. This provides some additional motivation and justification for Musa and okumoto to consider such a model.

Gamma IIDOS and NHPP models. The Gamma distribution has density

$$
f(x)=\gamma x^{\alpha-1} e^{-x / \beta}, 0<x,
$$


with $0<\alpha$ and $0<\beta$. If $0<\alpha \leq 1$, $\mathrm{f}(\cdot)$ is completely monotone and therefore by Theorem $6.6, \delta f(\cdot)$ can be the intensity of a failure-time process from some EOS model.

Another General Family of Models. The above Weibull and Gamma models are special cases of failure-time models with intensity

$$
\mathrm{m}(\mathrm{t})=\gamma \mathrm{x}^{\alpha-1} \exp \left(-(\mathrm{x} / \beta)^{\delta}\right), 0<\mathrm{t},
$$

where $0<\alpha \leq 1,0<\beta, 0<\delta \leq 1$. This function satisfies Theorem 6.6; thus the IIDOS process or NHPP with intensity $m(\cdot)$ can arise from an EOS model.

Geometric failure rates. The deterministic model

$$
\lambda_{\sim}=\left\{\lambda_{i}=\alpha \beta^{i}, i=1,2, \ldots\right\}
$$

with $0<\beta<1$ has been suggested by Nagel [21,22]. We now consider NHPP models for $\Lambda$ which will show an approximate geometric pattern. The analysis in Section 2 suggests an NHPP with

$$
\overline{\mathrm{L}}(\lambda)=\left\{\begin{array}{cl}
(\log \lambda-\log \alpha) / \log \beta & 0 \leq \lambda \leq \alpha \\
0 & \alpha \leq \lambda<\infty
\end{array}\right.
$$

because $\bar{L}^{-1}(i)=\alpha \beta^{i}, i=1,2, \ldots$. This NHPP $\Lambda$-process has intensity

$$
\ell(\lambda)=\left\{\begin{array}{cl}
-(\lambda \log \beta)^{-1} & 0 \leq \lambda \leq \alpha \\
0 & \alpha<\lambda<\infty .
\end{array}\right.
$$

The intensity and mean of the failure-time process are

$$
\begin{aligned}
& m(t)=\left(1-e^{-\alpha t}\right) /(t \log (1 / \beta)), 0 \leq t \\
& M(t)=\frac{1}{\log (1 / \beta)} \sum_{i=1}^{\infty} \frac{(-\alpha t)^{i}}{i \cdot i !}, 0 \leq t
\end{aligned}
$$


and by Theorem 3.4 the failure-time process $\{N(t), 0 \leq t\}$ is an NHPP.

Other methods of adding noise to the model of Equation (7.9) are suggested by considering

$$
-\log {\underset{\imath}{\lambda}}^{\lambda}=\left\{-\log \lambda_{i}=-\log \alpha-i \log \beta, i=1,2, \ldots\right\}
$$

which is a renewal process starting at $-\log \alpha$ and having constant interarrival times equal to $-\log \beta$. Using random i.i.d. interarrival times with mean $-\log \beta$ transforms this into a stochastic model. If i.i.d. exponential interarrival times are used, $-\log \Lambda$ is an HPP and $\AA$ is the same NHPP as above, with the intensity of Equation (7.11). If a general renewal process is used for $\log \Lambda$, the distribution of the failure-time process $\{N(t), 0 \leq t\}$ appears intractible.

The $\Gamma /$ EOS model with $\alpha=0$, i.e., the Musa-okumoto Logarithmic NHPP, may also serve as a model when the underlying failure rates are approximately geometric. Note that the intensity of Equation (7.11) and the intensity of the M-0 process, Equation (5.2), are both proportional to $t^{-1}$ for large $t$. This implies roughly the same pattern of failure rates among the later-occurring bugs, i.e., the bugs with small rates. More precisely, for the $M-0$ model

$$
\begin{aligned}
\overline{\mathrm{L}}(\lambda) & =\int_{\lambda}^{\infty} \ell(\mu) \mathrm{d} \mu=\int_{\lambda}^{\infty} \gamma \mu^{-1} \mathrm{e}^{-\beta \mu} \mathrm{d} \mu=\gamma \int_{1}^{\alpha} \nu^{-1} \mathrm{e}^{-\lambda \beta \nu} \mathrm{d} \nu \\
& =-\gamma\left(.5772157+\log \beta \lambda+\sum_{i=1}^{\infty} \frac{(-\beta \lambda)^{i}}{i \cdot i !}\right) .
\end{aligned}
$$

As $\lambda \rightarrow 0, \bar{L}(\lambda)$ behaves like $-\gamma \log \beta \lambda$; thus $\lambda_{i}=\bar{L}^{-1}(i)$ shows a geometric pattern for large $i$. This behavior in connection with the frequent occurrence of geometric rates in replicated run experiments $[8,21,22]$ suggests that the Musa-Okumoto Logarithmic NHPP will of ten be a good model; this is verified in their study using real data [20]. 
Logarithmic failure rates. Example 2 of Section 2, $\lambda=\left\{\lambda_{i}=\xi \log (\nu / i), 1 \leq i<[\nu]\right\}$, showed a logarithmic pattern. This can be generalized to

$$
\underset{\nu}{\lambda}=\left\{\lambda_{i}=\xi \log (\nu / i)^{1 / \alpha}, 1 \leq i \leq[\nu]\right\}
$$

where $0<\alpha$. These rates satisfy $\lambda_{i}=\overline{\mathrm{L}}^{-1}(i)$, where $\overline{\mathrm{L}}(\lambda)=\nu \exp \left(-(\lambda / \xi)^{\alpha}\right)$, which is proportional to a Weibull survival function. The stochastic version will have rates $\left\{\Lambda_{1}, \ldots, \Lambda_{\mathbf{n}}\right\}$ which are Weibu11 IIDOS, or NHPP with mean function $\left.M(t)=v\left(1-\exp (-\lambda / \xi)^{\alpha}\right)\right)$.

There are three different EOS models related to the Weibu11 distribution: In the above process the failure rates are Weibull distributed with shape parameter $0<\alpha<\infty$. In a previous example the failure times were Weibull distributed with shape parameter $0<\alpha<1$. Finally the Power Law NHPP is sometimes referred to as a Weibull NHPP because its intensity, $m(t)=\gamma t^{-\delta}$, equals the hazard function of a Weibull distribution with shape parameter $\alpha=1-\delta$, $0<\delta<1$.

Phillips law. Phillips (see [1]) observed that "the relative numbers of design errors having each possible rate was proportional to a particular inverse power of the rates." This is equivalent to $\ell(\lambda)=\gamma \lambda^{\alpha-1},-1<\alpha<0$, in our terminology, and gives the Power Law NHPP. In this case $\overline{\mathrm{L}}(\lambda)=(\gamma / \alpha) \lambda^{\alpha}$ and a deterministic model has $\lambda_{i}=(\alpha i / \gamma)^{1 / \alpha}, i=1,2, \ldots$ (Example 4 of Section 2). Note that the plot of $\left\{\left(\log i,-\log \lambda_{i}\right), i=1,2, \ldots\right\}$ is linear with slope $1 / \alpha,-1<1 / \alpha<0$. If $\lambda$ is used as a measure of the "size" of the design error, then we have a generalization of Zipf's Law [26, pp. 195-202]. Zipf's Law applies when the slope equals -1 , which corresponds to $\alpha=-1$; however, when $\alpha=-1, \lambda_{i}=\gamma / \alpha i$ and 
$\infty$

$\sum_{i=1} \lambda_{i}=\infty$ and a proper EOS model does not exist. Note, however, from Section 5 that a suitably normalized Power Law NHPP converges to an HPP as $\alpha \rightarrow-1$; so in this sense Zipf's Law for failure rates gives an HPP for failure times, i.e., no growth.

\section{Effect of previous debugging. A design flaw with failure rate $\lambda$} will remain undetected during $[0, \delta]$ with probability $e^{-\delta \lambda}$. Thus, if $\overline{\mathrm{L}}_{0}(\cdot)$ is the complementary mean function of the $\Lambda$-process for a program with no previous debugging, and if $\overline{\mathrm{L}}_{\delta}(\cdot)$ is for the $\Lambda$-process of bugs remaining after debugging for $\delta$ time units, then

$$
\mathrm{d} \overline{\mathrm{L}}_{\delta}(\lambda)=\mathrm{e}^{-\delta \lambda} \mathrm{d}_{0}(\lambda), \quad 0<\lambda
$$

Let $\mathrm{m}_{0}(\cdot)$ and $\mathrm{m}_{\delta}(\cdot)$ be the intensities of the respective failure time processes, then

$$
\begin{aligned}
\mathrm{m}_{\delta}(\mathrm{t}) & =-\int_{0}^{\infty} \lambda \mathrm{e}^{-\lambda t} \mathrm{~d} \overline{\mathrm{L}}_{\delta}(\lambda) \\
& =-\int_{0}^{\infty} \lambda \mathrm{e}^{-\lambda(\mathrm{t}+\delta)} \mathrm{d} \overline{\mathrm{L}}_{0}(\lambda) \\
& =\mathrm{m}_{0}(\mathrm{t}+\delta), 0 \leq \mathrm{t},
\end{aligned}
$$

(as one would expect). Since the choice of a time origin may be somewhat arbitrary, it is reassuring that models for different choices of origin are simply related as in Equations (7.15) and (7.16). In the $\Gamma /$ EOS models, the parameter $\beta$ could be interpreted as an indicator of something equivalent to previous debugging. The Generalized Power Law NHPP can be interpreted as a Power Law NHPP that has undergone previous debugging. A family of parametric models can be constructed using Equation (7.15) or (7.16) from a single EOS model. The family of $\Gamma / E O S$ models is closed under such construction; this should be considered a desirable property for a parametric family. Note that the NHPP of Equations (7.10) through (7.12) does not have this property. An enlarged 
family with

$$
\ell(\lambda)=\left\{\begin{array}{cc}
-(\lambda \log \beta)^{-1} \mathrm{e}^{-\delta \lambda} & 0 \leq \lambda \leq \alpha \\
0 & \alpha<\lambda<\infty
\end{array}\right.
$$

will have the property. But for large $\delta$, the truncation at $\alpha$ becomes negligible and we have the Musa-Okumoto Logarithmic NHPP again, of course.

Truncated models for $\Lambda$. Another modification that can be made to any model is truncation of the $\Lambda$-process at $\lambda_{\max }$ :

$$
\overline{\mathrm{L}}_{\text {trunc }}(\lambda)=\left\{\begin{array}{cl}
\overline{\mathrm{L}}(\lambda)-\overline{\mathrm{L}}\left(\lambda_{\text {max }}\right) & 0 \leq \lambda \leq \lambda_{\max } \\
0 & \lambda_{\max } \leq \lambda
\end{array} .\right.
$$

Also, inadmissible models can be made admissible by truncating; for example,

$$
\ell(\lambda)=\left\{\begin{array}{cl}
\gamma \lambda^{\alpha-1} & 0 \leq \lambda \leq \lambda_{\max } \\
0 & \lambda_{\max } \leq \lambda
\end{array}\right.
$$

is an admissible intensity for the $\Lambda$-process for $-1<\alpha<\infty$, but the nontruncated version is admissible only for $-1<\alpha<0$.

Superposition of models. The superposition of two or more EOS models is again an EOS model. A program might have two main subparts and an interface between them, a11 written or designed by different people. Bugs can be identified as belonging to one of the three parts; this gives three failure-time processes: $\left\{N_{1}(t), 0 \leq t\right\},\left\{N_{2}(t), 0 \leq t\right\}$ and $\left\{N_{3}(t), 0 \leq t\right\}$. If each of the three is an EOS process then $\mathrm{N}_{1}+\mathrm{N}_{2}+\mathrm{N}_{3}$ is a failure-time process of an EOS model. In general, for $\underset{\imath}{\lambda}=\left\{\lambda_{1}, \lambda_{2}, \ldots \lambda_{n}\right\}$, let $N_{i}(t)=1_{[0, t]}\left(x_{i}\right), i=1,2, \ldots, n ;$ then 
$N(t)=\sum_{i=1}^{n} N_{i}(t)=\sum_{i=1}^{n} 1_{[0, t]}\left(X_{i}\right), 0<t$, is the general deterministic EOS model of Section 2. Applying Theorem 4.9 gives that superposition of DET/EOS models, each consisting of one bug, are dense among all DET/EOS models. Thus any reasonably rich family of EOS models that is closed under superposition of its members and limits of its members will contain all EOS models. The $\Gamma /$ EOS family is not closed under superposition, but it may still be a family that is rich enough for many applications.

Interfailure time models. Interfailure time models assume that successive interfailure times are independent (but not identically distributed). The Littlewood-Verra11 [18] model is an example. It can be shown that the Jelinsky-Moranda model has independent interfailure times. It can also be shown that any EOS model with three or more bugs and nonconstant failure rates cannot have independent interfailure times. Thus, except for the overlapping J-M model, the EOS models and interfailure time models are distinct classes of models. Nevertheless, certain EOS models do suggest possible interfailure time models. For example, the Musa-Okumoto Logarithmic NHPP has $M(t)=\gamma \log (t / \beta+1)$, so the ith failure will occur somewhere around $s_{i}=M^{-1}(i)=\beta(\exp (i / \gamma)-1)$. They show that the interfailure time $S_{i+1}-S_{i}$ has a Pareto distribution conditional on $\mathrm{s}_{\mathbf{i}}=\mathbf{s}$ :

$$
\left.P\left(S_{i+1}-S_{i}>t \mid s_{i}=s\right)=(t / s+\beta)+1\right)^{-\gamma}, 0 \leq t
$$

Since $S_{i}$ is approximately equal to $s_{i}$, then

$$
\left.P\left(S_{i+1}-S_{i}>t\right) \approx\left(t / s_{i}+\beta\right)+1\right)^{-\gamma}, 0 \leq t,
$$

and we postulate an approximate interfailure time model with Pareto interfailure times; the parameters of the ith interfailure time are $\gamma$ and 


$$
\psi_{i}=s_{i}+\beta=\beta(\exp (i / \gamma)-1)+\beta=\beta e^{i / \gamma}, i=1,2, \ldots
$$

The Littlewood-Verrall is exactly such a model, having independent Pareto interfailure times:

$$
P\left(S_{i+1}-S_{i}>t\right)=\left(t / \psi_{i}+1\right)^{-\alpha}
$$

with $\psi_{i}$ increasing. They used $\psi_{i}=\beta_{i}+\beta_{2} i$ and $\psi_{i}=\beta_{1}+\beta_{2} i^{2}$. The above admittedly rough analysis suggests that $\psi_{i}=\beta_{1} \beta_{2} i$ be tried.

8. Inference and Prediction

Software reliability growth models are useful in assessing software reliability; but creating models is only one step of the process. A typical scenario involves observing failures and correcting design flaws at times $s_{1}, s_{2}, \ldots, s_{n}(t)$ during $[0, t]$ and then trying to predict behavior during $[t, \infty)$; quantities that are of interest include the current failure rate, the distribution of time until the next failure, the number of failures over a finite horizon, etc. Littlewood (et al, Evaluation of Competing Software Reliability Predictions, Centre for Software Reliability Research Report, The City University, London, March 1985) describes a "prediction system," which involves three components:

(i) Probability models that completely specify the distribution of failure times $\left(s_{1}, s_{2}, \ldots\right)$;

(ii) An inference procedure for picking a specific single model for particular observed data $\left(s_{1}, s_{2}, \ldots, s_{n(t)}\right)$;

(iii) A prediction procedure that combines (i) and (ii) to give probability statements about future failure times $\left(s_{N(t)+1}, s_{N(t)+2}, \ldots\right)$. 
Our focus now is on part (i) of Littlewood's Triad; this paper's main concern is a particular class of probability models: Eos models. Nevertheless there are some observations pertaining to inference and prediction for EOS models that are worth making at this time. We shall make these briefly; more extensive study will appear in future research papers.

The family of EOS models is very rich. The possible patterns of underlying failure rates are unlimited. The above inference and prediction steps will be easier if the class of possible models can be restricted a priori, i.e., before testing or, ideally, before the program is written. After many controlled software experiments are conducted and a large amount of field data are collected it may be possible to conclude that particular types of software tend to exhibit characteristic patterns for the failure rates. A geometric pattern $\lambda_{\nu}=\left\{\alpha \beta^{i}, 0<i\right\}$ is emerging in the programs studied in certain replicated run experiments $[8,21,22]$. Phillips observed a different pattern in some IBM software [1]. Perhaps various software metrics [11] can be used to discriminate between classes of software that show different patterns in their underlying failure rates. A Bayesian analysis based on an a priori distribution over the entire class of EOS models would be very desirable; it is not clear at this time how to describe such a distribution. The point is that the richness and generality of all possible EOS models seem to suggest the need for a capability to base reliability predictions on more than just observation of past failures of the specific program in question.

On the other hand, the $\Gamma / E O S$ family of models may be rich enough to represent adequately the entire family of EOS models. Further study is required to confirm this, but it does look promising. The $\Gamma / E O S$ family includes both the geometric pattern and the pattern described by Phillips. It also includes as special cases several well-known models. Possible advantages to considering the single family parameterized by $(\alpha, \beta, \gamma)$ instead of considering the separate models should be explored. A Bayesian approach with this three-dimensional parametric family should be pursued. 
The richness of the EOS family of models suggests that a nonparametric approach to inference and prediction be tried. The complete monotonicity properties of Section 6 and Theorem 4.7 reveal an approach: Estimate $M(t), 0 \leq t$, with the curve satisfying Theorem 6.7 , which is "closest" to the observed realization of $\{N(t), 0 \leq t\}$. Or one can estimate the intensity of $\{N(t), 0 \leq t\}$ from the observed sample path using any of several nonparametric estimates and then find the "closest" function that satisfies Theorem 6.6 for an improved estimate. The first step in this direction has been taken by Campbell and ott [4]; they used isotonic regression methods to estimate the intensity function of EOS failure processes. Miller and Sofer [19] explore the more general problem of regression with completely monotone functions.

The nondistinguishability between NHPP and IIDOS models discussed in Section 4 has some ramifications for inference and prediction. If two alternatives $\mathrm{H}_{1}$ and $\mathrm{H}_{2}$ are statistically indistinguishable given the data, the question of "goodness-of-fit" is moot. However, in the case of NHPP versus IIDOS inference will differ: the likelihood functions in the two cases are different and so are admissible parameter values, e.g., $M(\infty)$ must be integer-valued for IIDOS models. but not for NHPP models. Thus we may get different predictions using the two alternatives to which we are indifferent as far as any goodness-of-fit criteria are concerned. Since "quality of prediction" is of primary importance, the indifference between NHPP and IIDOS models in the first stage (i) of the prediction system may not carry over to the third stage (iii).

\section{Conclusions}

This paper has presented EOS models and some of their properties. The idea of nonidentically distributed independent Exponential random variables is, of course, not new. However, when doubly stochastic EOS models are considered and when only a single sample path is observed, some interesting properties follow that are relevant to the study of software reliability growth.

Exponential Order Statistic models are useful for several reasons. The structure of the models reflects to some extent the actual physical 
failure process: the parameter $\lambda$ has a physical intepretation, and the doubly stochastic models distinguish between randomness of the failure rates and randomness in the usage environment. This may eventually allow deeper penetration into the mysteries of real failure processes; EOS models seem to be natural models for replicated-run software debugging experiments. The fact that several well-known software reliability growth models can be derived as special cases of EOS models is useful because the alternative derivation provides additional support for the validity of those models. Finally, the EOS paradigm is a framework within which new parametric families of reliability growth models can be identified (e.g., Section 7).

A general adoption of EOS models leads to several new possibilities and points of view concerning inference and prediction. The mean $M(\cdot)$ and intensity $\mathrm{m}(\cdot)$ of the failure process play a central role, as does the process of failure rates, $\underset{\sim}{\Lambda}$; the relationship between these quantities is that of a Laplace transform, a connection which might be exploitable. The $\Gamma / E O S$ model unifies several previously unrelated models; a unified approach to inference and prediction for these models based on the r/EOS model should be useful. The richness of the family of EOS models and the possibility of superposition of failure processes tend to challenge the ability of a parametric family to model all possibilities adequately. Nonparametric approaches can be based on complete monotonicity properties. Development of a Bayesian analysis encompassing the entire EOS family is worth pursuing. Lastly, the EOS family gives a diverse set of models from which data can be simulated in a systematic way and used as test data for evaluating various proposed inference and prediction methods.

It is hoped that this paper will stimulate interest that will lead to further use and investigation of these models. 
APPENDIX

This appendix contains background information, proofs and additional discussion. Ross $[23,24]$ provides a good introduction to the necessary stochastic processes, especially the Poisson process. Karlin and Taylor [13, Chapter 13] provide an excellent discussion of uniform order statistic processes, homogeneous Poisson processes and their relationship to one another. Cinlar [5] gives an excellent discussion of Poisson processes, especially the nonhomogeneous (nonstationary) Poisson process, which he shows to be a transform in time of a homogeneous Poisson process [5; Chapter 4, Section 7]; this leads to generalization of the results in Karlin and Taylor to nonuniform order statistics and nonhomogeneous Poisson processes.

In Section 2 a proper DEI/EOS model is defined as one for which $\infty$ $\sum_{i=1} \lambda_{i}<\infty$. Recall that $x_{i} \sim$ Exponential $\left(\lambda_{i}\right), i=1,2, \ldots$, are independent random variables. The event $\{N(t)<\infty\}$ belongs to the $\sigma$-field generated by $\left\{x_{1}, x_{2}, \ldots\right\}$, i.e., occurrence or nonoccurrence is completely determined by the values taken by $\mathrm{x}_{1}, \mathrm{x}_{2}, \ldots$. Furthermore, the event $\{\mathrm{N}(\mathrm{t})<\infty\}$ is independent of $\left\{\mathrm{x}_{1}, \mathrm{x}_{2}, \ldots, \mathrm{x}_{\mathrm{n}}\right\}$ for each $n, n<\infty$. Thus by the Zero-One Law for Tail Events [9, p. 124], either $\mathrm{P}\{\mathrm{N}(\mathrm{t})<\infty\}=0$ or $\mathrm{P}\{\mathrm{N}(\mathrm{t})<\infty\}=1$. When $\Sigma \lambda_{i}<\infty$, $\operatorname{minx}_{i} \sim$ Exponential $\left(\Sigma \lambda_{i}\right)$; thus $\mathrm{P}\{\mathrm{N}(\mathrm{t})=0\}=\exp \left(-\Sigma \lambda_{i}\right)>0$ and the latter possibility holds: $\mathrm{P}\{\mathrm{N}(\mathrm{t})<\infty\}=1$. Conversely, if $\mathrm{P}\{\mathrm{N}(\mathrm{t})<\infty\}=1$ then for some $n<\infty, P\{N(t)=n\}>0$, which implies that there is at least one subset of the integers $\left\{i_{1}, i_{2}, \ldots, i_{n}\right\}=I_{n}(t)$ such that $P\left(\left\{X_{i} \leq t\right.\right.$, i $\left.\varepsilon I_{n}(t)\right\} \cap\left\{X_{i}>t\right.$, $\left.\left.i \notin I_{n}(t)\right\}\right)>0$, which in turn implies $P\left(X_{i}>t, i \notin I_{n}(t)\right)>0$ which equals $\underset{i \notin I_{n}(t)}{\exp \left(-\sum_{i}\right)} \lambda_{i}$ Thus 
if $\lambda_{i}<\infty$, for $i \in I_{n}(t)$, we get $\sum_{i=1}^{\infty} \lambda_{i}<\infty$. This proves (under the assumption that $\left.\lambda_{i}<\infty, i \geq 1\right)$ that $\{N(t), t<\infty\}$ is nondegenerate iff $\sum_{i=1}^{\infty} \lambda_{i}<\infty$. If $\lambda_{i}=\infty$ for $i=1, \ldots, k$ and $\lambda_{i}<\infty$ for $i>k$, then $N(0)=k$ and $N(t)<\infty$ with probability one iff $\sum_{i=k+1}^{\infty} \lambda_{i}<\infty$

The Exponential approximation to the Geometric mentioned in Section 2 is well known. The formal statement in terms of a limit theorem is: Let $X_{n}, n=1,2, \ldots$, be a sequence of Geometric random variables with parameters $p_{n}, n=1,2, \ldots$, respectively, i.e., $P\left(x_{n}>i\right)=\left(1-p_{n}\right)^{i}$, $i=0,1,2, \ldots$. If $\lim _{n \rightarrow \infty} p_{n}=0$, then $\lim _{n \rightarrow \infty} P\left(p_{n} x_{n}>y\right)=e^{-y}$. Thus for small $p$ the Exponential distribution can be used as an approximation to the Geometric distribution.

Another situation wherein the time until failure is approximately Exponential involves regenerative processes. (See Cinlar [5] or Ross [24] for background.) Successive inputs to a program may not be independent but may instead exhibit dependence; for example, the sequence of inputs could be a random walk on the space of all possible inputs. More generally, the sequence of inputs could be a regenerative process. Such processes have a structure which consists of a sequence of independent identically distributed sojourns from a single state, which is called a regeneration point. If on any given sojourn the probability of experiencing the failure is $\mathrm{p}$ and the failure first occurs on sojourn $\mathrm{N}$, then $P\{N=n\}=p(1-p)^{n-1}, n=1,2, \ldots$. The duration of sojourns 1 through $\mathrm{N}-1$ will be $\mathrm{i} . \mathrm{i} . \mathrm{d}$. random variables $\mathrm{T}_{1}, \mathrm{~T}_{2}, \ldots, \mathrm{T}_{\mathrm{N}-1}$. If ET $<\infty$, it can be shown using Laplace transform arguments that, as $p \rightarrow 0$, the distribution of $p \sum_{i=1}^{n-1} T_{i}$ converges to an Exponential 
distribution with mean ET. Thus if the duration in the Nth sojourn until failure is negligible compared to $\sum_{i=1}^{n-1} T_{i}$ and $p$ is small, the distribution of time until failure will be approximately Exponential.

Theorems 3.1 and 3.2 are well known. Theorem 3.1 deals with transforming i.i.d. uniform order statistics into i.i.d. order statistics of an arbitrary probability distribution using the "inverse probability transform;" see Law and Kelton [15, pp. 242-247] or Schmeiser [25]. Theorem 3.2 deals with transforming an HPP into an NHPP; see Cinlar [5, pp. 94-101] for a proof.

Proof of Theorem 3.3: Consider the unordered failure rates $\left(\Lambda_{1}, \Lambda_{2}, \ldots, \Lambda_{n}\right)$ where $n=L(\infty): P\left(\Lambda_{1} \leq \lambda_{1}, \Lambda_{2} \leq \lambda_{2}, \ldots, \Lambda_{n} \leq \lambda_{n}\right)=$ $\prod_{i=1}^{n} L\left(\lambda_{i}\right) / n$. Let $T_{i}\left(\Lambda_{i}\right)$ be an exponential random variable with rate $\Lambda_{i}$; thus $P\left(T_{i}\left(\Lambda_{i}\right)<t\right)=\int_{0}^{\infty} P\left(T_{i}\left(\Lambda_{i}\right)<t \mid \Lambda_{i}=\lambda\right) d L(\lambda) / n=\int_{0}^{\infty}\left(1-e^{-\lambda t}\right) d L(\lambda) / n$. The $\left(\mathrm{T}_{1}\left(\Lambda_{1}\right), \ldots, \mathrm{T}_{\mathrm{n}}\left(\Lambda_{\mathrm{n}}\right)\right)$ are independent because the $\left(\Lambda_{1}, \ldots, \Lambda_{\mathrm{n}}\right)$ are independent; thus we have a random sample of size $\mathrm{n}$ from cdf $F(t)=\int_{0}^{\infty}\left(1-e^{-\lambda t}\right) d L(\lambda) / n$ and the ordered $T_{i}\left(\Lambda_{i}\right)$ 's are an IIDOs process. The mean function is $M(t)=\operatorname{EN}(t)=E\left(\sum_{i=1}^{n} 1_{[0, t]}\left(T_{i}\left(\Lambda_{i}\right)\right)\right)$

$=\sum_{i=1}^{n} P\left(T\left(\Lambda_{i}\right)<t\right)=\int_{0}^{\infty}\left(1-e^{-\lambda t}\right) d L(\lambda)$.

Proof of Theorem 3.4: Consider the Poisson process of (ordered) failure rates: $\Lambda_{1} \geq \Lambda_{2} \geq \Lambda_{3} \geq \ldots$. This process has complementary cumulative mean function $\overline{\mathrm{L}}(\cdot)$. Now consider $0=\mathrm{t}_{0}<\mathrm{t}_{1}<\mathrm{t}_{2}<\ldots<\mathrm{t}_{\mathrm{k}}$ and split the above process into $k$ processes: $\mathrm{N}_{1}, \mathrm{~N}_{2}, \ldots, \mathrm{N}_{\mathrm{k}}$. 
If $\Lambda_{i}=\lambda_{i}$ then this failure rate is put into the $j$ th process with probability $e^{-\lambda_{i} t_{j-1}}-e^{-\lambda_{i} t_{j}}, j=1,2, \ldots, k$. By well-known splitting theorem for NHPP's, $\mathrm{N}_{1}, \mathrm{~N}_{2}, \ldots, \mathrm{N}_{\mathrm{k}}$ are independent Poisson processes with $E N_{i}(\infty)=\int_{0}^{\infty}\left(e^{-\lambda t} j-1-e^{-\lambda t} j\right)(-d \bar{L}(\lambda))$. But note that the splitting is equivalent to observing $T_{i}\left(\Lambda_{i}\right), i=1,2, \ldots$ : if $\Lambda_{i}=\lambda_{i}$ then $P\left(t_{j-1}<T_{i}\left(\Lambda_{i}\right) \leq t_{j} \mid \Lambda_{i}=\lambda_{i}\right)=e^{-\lambda_{i} t_{j-1}}-e^{-\lambda_{i} t_{j}}$. So $\left.N_{i}(\infty)=\sum_{i=1}^{\infty} 1 t_{j-1}, t_{j}\right)\left(T_{i}\left(\Lambda_{i}\right)\right), j=1,2, \ldots k$. But from above, $\mathrm{N}_{j}(\infty), j=1,2, \ldots k$, are independent Poisson random variables with $E\left(\sum_{j=1}^{\ell} N_{j}(\infty)\right)=\int_{0}^{\infty}\left(1-e^{-\lambda t} j\right)(-d \bar{L}(\lambda))$. Thus $\left\{T_{i}\left(\Lambda_{i}\right), i=1,2, \ldots\right\}$ satisfy the postulates for an NHPP with mean function $M(t)=\int_{0}^{\infty}\left(1-e^{-\lambda t}\right)(-d \bar{L}(\lambda))$

Theorems 4.1 through 4.5 are generalizations of relationships between uniform order statistics and homogeneous Poisson processes (see Karlin and Taylor [13]). These relationships extend to order statistics from general distribution and NHPP's using the timetransformation which changes an HPP into an NHPP (see Cinlar [5]). Theorem 4.6 is somewhat more general: from Theorems 4.2 and 4.3 $\left\{N_{1}(t), 0 \leq t \leq s \mid N_{1}(s)=n\right\}$ and $\left\{N_{2}(t), 0 \leq t \leq s \mid N_{2}(s)=n\right\}$ are identically distributed. Since $\mathrm{T}_{1}$ and $\mathrm{T}_{2}$ are identical and the occurrence of the events $\left\{\mathrm{T}_{1}=\mathrm{s}\right\}$ and $\left\{\mathrm{T}_{2}=\mathrm{s}\right\}$ just depends on the behavior of their respective processes during $[0, s]$, the conditional processes in the statement of Theorem 4.6 will be identically distributed. 
Theorem 4.7 follows from various forms of the strong law of large numbers. Theorem 4.8 follows from various forms of multivariate central 1imit theorens. For an HPP the limit is a Brownian motion; for the NHPP with mean function $M(t)$ the 1imiting Gaussian process has covariance function $\operatorname{Cov}(s, t)=M(\min (s, t))$. For a process of uniform order statistics the limit is a Brownian bridge; for an IIDos process with mean function $M(t)$ the limiting Gaussian process has covariance function $\operatorname{Cov}(s, t)=M(s)(M(\infty)-M(t)) / M(\infty), s<t ; c f$. Billingsley [2, Theorem 16.4]. For the DET/EOS process we note that

$$
\begin{aligned}
\operatorname{Var}\left(N_{k}(t) / \sqrt{k}\right) & =k^{-1} \sum_{i} \operatorname{Var}\left({ }^{1}[0, t]\left(T_{i}\left(\lambda_{i}\right)\right)\right) \\
& =k^{-1} \sum_{i}\left(\left(1-e^{-\lambda_{i} t}\right)\left(1-\left(1-e^{-\lambda_{i} t}\right)\right)^{2}+e^{-\lambda_{i} t}\left(0-\left(1-e^{-\lambda_{i} t}\right)^{2}\right)\right. \\
& =k^{-1} \sum_{i} e^{-\lambda_{i} t}\left(1-e^{-\lambda_{i} t}\right) \\
& =k^{-1} \sum_{i} \exp \left(-\bar{L}^{-1}(i / k) t\right)\left(1-\exp \left(-\bar{L}^{-1}(i / k) t\right)\right) \\
& =k^{-1} \sum_{i} \exp \left(-\bar{L}^{-1}(i / k)\right)^{t}\left(1-\exp \left(-\bar{L}^{-1}(i / k)\right)^{t}\right) .
\end{aligned}
$$

This is a Riemann sum, which converges to

$$
V(t)=\int_{0}^{\bar{L}(0)} \exp \left(-\bar{L}^{-1}(x)\right)^{t}\left(1-\exp \left(-\bar{L}^{-1}(x)\right)^{t}\right) d x
$$

as $k \rightarrow \infty$. Similarly the limiting covariance is

$$
\operatorname{Cov}(s, t)=\int_{0}^{\bar{L}(0)} \exp \left(-\bar{L}^{-1}(x)\right)^{s}\left(1-\exp \left(-\bar{L}^{-1}(x)\right)^{t} d x,\right.
$$


$s<t$. As an example (which is tractible) consider case 2 of Section 2: $\overline{\mathrm{L}}(\lambda)=\nu \exp (-\lambda / \xi) ; \overline{\mathrm{L}}^{-1}(\mathrm{x})=-\xi \log (\mathrm{x} / \nu)$, so the limiting covariance is

$$
\begin{aligned}
\operatorname{Cov}_{0}(s, t) & =\int_{0}^{\nu}(x / v)^{\xi s}\left(1-(x / \nu)^{\xi t}\right) d x \\
& =v \int_{0}^{1} u^{\xi s}\left(1-u^{\xi t}\right) d u \\
& =\frac{v \xi t}{(\xi s+1)(\xi s+\xi t+1)}
\end{aligned}
$$

The covariance of the IIDOS process with the same $\overline{\mathrm{L}}(\cdot)$ is

$$
\begin{aligned}
\operatorname{Cov}_{1}(s, t) & =\int_{0}^{\infty}\left(1-e^{-\lambda s}\right) d L(\lambda) \int_{0}^{\infty} e^{-\lambda t} d L(\lambda) / L(\infty) \\
& =\nu\left(1-\frac{\xi s}{\xi s+1}\right) \frac{\xi t}{\xi t+1} \\
& =\frac{\nu \xi t}{(\xi s+1)(\xi t+1)} \quad, \quad s<t .
\end{aligned}
$$

Note that $\operatorname{Cov}_{1}(s, t)>\operatorname{Cov}_{0}(s, t)$, as expected.

Proof of Theorem 4.9: Let $x_{i}, i=1,2, \ldots$, be independent Exponential random variables with rates $\lambda_{i}, i=1,2, \ldots$, respectively. Define $N_{k}(t)=\sum_{i=1}^{k} 1_{[0, t]}\left(x_{i}\right), 0 \leq t, k=1,2, \ldots ;$ these processes are all defined on the same probability space and satisfy $N_{k}(t) \leq N_{k+1}(t)$, $0 \leq t, k=1,2, \ldots$, with probability one. By monotone convergence, $\lim _{k \rightarrow \infty} i_{k}(t)$ exists (possibly $\infty$ ) with probability one. The limit $k \rightarrow \infty$

$\sum_{i=1}^{\infty} 1_{[0, t]}\left(X_{i}\right)$ must be a nondegenerate process because $\Sigma \lambda_{i}<\infty$. Thus we have convergence with probability one to a DET/EOS process with an 
infinite number of events.

Proof of Theorem 4.10: The finite dimensional joint distributions of an IIDOS process with mean function $M_{k}(\cdot)$ are of the form

$$
\begin{aligned}
& P\left\{N_{k}\left(t_{1}\right)=n_{1}, N_{k}\left(t_{2}\right)=n_{2}, \ldots, N_{k}\left(t_{m}\right)=n_{m}\right\} \\
& =\left(\begin{array}{c}
M_{k}(\infty) \\
i_{1} i_{2} \ldots i_{m}
\end{array}\right) \underset{j=1}{m}\left(F_{k}\left(t_{j}\right)-F_{k}\left(t_{j-1}\right)\right)^{i} j\left(1-F_{k}\left(t_{m}\right)\right)^{M_{k}(\infty)-n_{m}},
\end{aligned}
$$

where $0=t_{0}<t_{1}<t_{2}<\ldots<t_{m}<\infty, F_{k}(\cdot)=M_{k}(\cdot) / M_{k}(\infty)$, $i_{j}=n_{j}-n_{j-1}, j=1,2, \ldots m$, and

$$
\left(\begin{array}{c}
M_{k}(\infty) \\
i_{1} i_{2} \ldots i_{m}
\end{array}\right)=\frac{M_{k}(\infty) !}{i_{1} ! i_{2} ! \ldots i_{m} !\left(M_{k}(\infty)-\sum_{1}^{m} i_{m}\right) !}
$$

If $\lim _{k \rightarrow \infty} M_{k}(t)=M_{\infty}(t)$ such that $M_{\infty}(\infty)<\infty$, then, as $k \rightarrow \infty$, the above joint distribution converges to

$$
\left(\begin{array}{c}
M_{\infty}(\infty) \\
i_{1} i_{2} \cdots i_{m}
\end{array}\right) \prod_{j=1}^{m}\left(F_{\infty}\left(t_{j}\right)-F_{\infty}\left(t_{j-1}\right)\right)^{i} j\left(1-F_{\infty}\left(t_{m}\right)\right)^{M_{\infty}(\infty)-n_{m}},
$$

the joint distribution of an IIDOS process with mean function $\mathrm{M}_{\infty}(\cdot)$. If $\lim _{k \rightarrow \infty} M_{k}(t)=M_{\infty}(t)$ such that $M_{\infty}(\infty)=\infty$ then, as $k \rightarrow \infty$, the above joint distribution may be rewritten as 


$$
\begin{aligned}
& \left(M_{k}(\infty)\right)^{-n_{m}}\left(\begin{array}{c}
M_{k}(\infty) \\
i_{1} i_{2} \cdots i_{m}
\end{array}\right) \underset{j=1}{m}\left(M_{k}\left(t_{j}\right)-M_{k}\left(t_{j-1}\right)^{i_{j}}\left(1-M_{k}\left(t_{m}\right) / M_{k}(\infty)\right)\right. \\
& \prod_{j=1}^{m} \frac{\left(M_{\infty}\left(t_{j}\right)-M_{\infty}\left(t_{j-1}\right)\right)^{i}}{i_{j} !} \lim _{k \rightarrow \infty} \frac{M_{k}(\infty) !}{M_{k}(\infty)^{M_{m}}\left(M_{k}(\infty)-n_{m}\right) !}\left(1-\frac{M_{k}\left(t_{m}\right)}{M_{k}(\infty)}\right)^{M_{k}(\infty)-n_{m}}
\end{aligned}
$$

but

$$
\lim _{k \rightarrow \infty} \frac{M_{k}(\infty) !}{M_{k}(\infty)} \frac{n_{m}\left(M_{k}(\infty)-n_{m}\right) !}{n}=1
$$

and

$$
\lim _{k \rightarrow \infty}\left(1-\frac{M_{k}\left(t_{m}\right)}{M_{k}(\infty)}\right)^{M_{k}(\infty)-n_{m}}=\exp \left(-M_{\infty}\left(t_{m}\right)\right),
$$

so the joint distribution converges to

$$
\begin{aligned}
& \prod_{j=1}^{m} \frac{\left(M_{\infty}\left(t_{j}\right)-M_{\infty}\left(t_{j-1}\right)^{i}\right.}{i_{j} !} \exp \left(-M_{\infty}\left(t_{m}\right)\right) \\
= & \prod_{j=1}^{m}\left(\exp \left(-\left(M_{\infty}\left(t_{j}\right)-M_{\infty}\left(t_{j-1}\right)\right)\right) \frac{\left(M_{\infty}\left(t_{j}\right)-M_{\infty}\left(t_{j-1}\right)\right)^{i}}{i_{j} !}\right),
\end{aligned}
$$

the joint distribution of an NHPP with mean function $M_{\infty}(\cdot)$, cf. Miller [18].

Proof of Theorem 4.11: It can be shown as above that the finite dimensional joint distributions of a sequence of NHPP's with mean functions $M_{k}(\cdot)$ converge to those of an NHPP with mean function $M_{\infty}(\cdot)$ as $k \rightarrow \infty$. 
Proof of Theorem 6.1: Since $\overline{\mathrm{L}}(\lambda)$ is integer-valued, $\int_{0}^{\infty} \overline{\mathrm{L}}(\lambda) \mathrm{d} \lambda<\infty$ if and only if $\lim _{\lambda \rightarrow \infty} \overline{\mathrm{L}}(\lambda)=0$ and $\int_{0}^{\mu} \overline{\mathrm{L}}(\lambda) \mathrm{d} \lambda<\infty$. $\operatorname{But} \int_{0}^{\infty} \bar{L}(\lambda) \mathrm{d} \lambda=\sum_{i=1}^{\infty} \lambda_{i}$, where $\lambda_{i}=\bar{L}^{-1}(i)$.

Proof of Theorem 6.2: Since $\Lambda_{1}$ is the largest rate, $P\left(\sum_{i=1}^{n} \Lambda_{i}<\infty\right)=1$ if and only if $P\left(\Lambda_{i}<\infty\right)=1 ; P\left(\Lambda_{i}<\lambda\right)=$ $((\overline{\mathrm{L}}(0)-\overline{\mathrm{L}}(\lambda)) / \overline{\mathrm{L}}(0))^{\mathrm{n}}$, so $\mathrm{P}\left(\Lambda_{i}<\infty\right)=1$ if and only if $\underset{\lambda \rightarrow \infty}{\lim } \overline{\mathrm{L}}(\lambda)=0$.

Proof of Theorem 6.3: The largest rate $\Lambda_{1}$ is finite if and only if $\lim _{\lambda \rightarrow \infty} \overline{\mathrm{L}}(\lambda)=0$ (if $\mathrm{Y}_{1} \sim$ Exponential (1), then $\Lambda_{1}=\overline{\mathrm{L}}^{-1}\left(\mathrm{Y}_{1}\right)<\infty$ ). By Theorem 6.1 the conditions $\lim _{\lambda \rightarrow \infty} \bar{L}(\lambda)=0$ and $\int_{0}^{\mu} \bar{L}(\lambda) d \lambda<\infty, 0<\mu<\infty$, are equivalent to $\sum_{i=1}^{\infty} \lambda_{i}<\infty$, where $\lambda_{i}=\bar{L}^{-1}(i), i=1,2, \ldots$. Thus it suffices to show that $\mathrm{P}\left(\Sigma^{*} \Lambda_{i}<\infty\right)=1$, where $\Sigma^{*}$ is over all $\Lambda_{i} \leq \lambda_{1}$, if and only if $\sum_{i=1}^{\infty} \lambda_{i}<\infty$. Let $N_{i}=\sum_{j=1}^{\infty}{ }^{1}\left(\lambda_{i=1}, \lambda_{i}\right]\left(\Lambda_{j}\right), i=1,2, \ldots$, these are i.i.d. Poisson random variables with mean 1 ; then $\Sigma^{*} \Lambda_{i} \leq \sum_{i=1}^{\infty} N_{i} \lambda_{i}$ and $E\left(\Sigma{ }^{*} \Lambda_{i}\right) \leq E\left(\sum_{i=1}^{\infty} N_{i} \lambda_{i}\right)=\sum_{i=1}^{\infty} E N_{i} \lambda_{i}=\sum_{i=1}^{\infty} \lambda_{i}$. Therefore $\Sigma \lambda_{i}<\infty$ implies $E\left(\Sigma * \Lambda_{i}\right)<\infty$, which in turn implies $P\left(\Sigma \Lambda_{i}<\infty\right)=1$. Conversely, suppose that $\sum_{i=1}^{\infty} \lambda_{i}=\infty$. We shall randomly select a subset $S$ of $\lambda_{\sim}^{\lambda}$ : If $N_{i} \geq 1, \lambda_{i+1}$ : belongs to $S$, if $N_{i}=0, \lambda_{i+1}$ does not. Thus each $\lambda_{i+1}, i=1,2, \ldots$, is randomly and independently selected with 
probability $1-\mathrm{e}^{-1}>1 / 2$; thus $\sum_{\mathrm{S}} \lambda_{i+1}=\infty$ also. Note, however, that for each $\lambda_{i+1} \varepsilon S$ there exists a $\Lambda_{j(i)}$ such that $\lambda_{i+1}<\Lambda_{j(i)} \leq \lambda_{i}$. Therefore $\sum \lambda_{i+1}<\sum \Lambda_{j(i)}$ and we have a subset of $\Lambda$ whose sum diverges with probability one.

Proof of Theorem 6.4: There exists $\mu, 0<\mu<\infty$, such that $m(t)=-\int_{0}^{\infty} \lambda e^{-\lambda t} d \bar{L}(\lambda)<-\int_{0}^{\mu} \lambda d \bar{L}(\lambda)-\int_{\mu}^{\infty} d \bar{L}(\lambda)=-\int_{0}^{\mu} \lambda d \bar{L}(\lambda)+\bar{L}(\mu)$. Integration by parts gives $-\int_{0}^{\mu} \lambda \mathrm{d} \overline{\mathrm{L}}(\lambda)=\int_{0}^{j} \overline{\mathrm{L}}(\lambda) \mathrm{d} \lambda-\mu \mathrm{L}(\mu)+\lim _{\lambda \rightarrow 0} \lambda \overline{\mathrm{L}}(\lambda)$. But $\mu \mathrm{L}(\mu)<\int_{0}^{\mu} \overline{\mathrm{L}}(\lambda) \mathrm{d} \lambda$, which in turn is finite by the admissibility assumption. Thus $-\int_{0}^{\mu} \lambda d \overline{\mathrm{L}}(\lambda)<\infty$, giving $\mathrm{m}(\mathrm{t})<\infty$. Similarly, $M(t)<-\int_{0}^{1} \lambda d \bar{L}(\lambda)-\int_{1}^{\infty} d \bar{L}(\lambda)=-\int_{0}^{1} \lambda d \bar{L}(\lambda)+\bar{L}(1)$, which is finite by admissibility of $\overline{\mathrm{L}}(\cdot)$. Conversely, suppose $M(t)=$ $-\int_{0}^{\infty}\left(1-e^{-\lambda t}\right) d \bar{L}(\lambda)<\infty$ and $\lim _{t \rightarrow 0} M(t)=0$. But $1-e^{-\lambda t} \geq 1 / 2$ for $\log 2 / t \leq \lambda<\infty$, which implies $M(t)>-\int_{(\log 2) / t}^{\infty} 1 / 2 \mathrm{~d} \overline{\mathrm{L}}(\lambda)=$ $1 / 2 \overline{\mathrm{L}}(\log 2 / \mathrm{t})$.

Thus $\lim _{t \rightarrow 0} M(t)=0$ implies $\lim _{\lambda \rightarrow \infty} \bar{L}(\lambda)=0$ and we have admissibility. Proof of Theorem 6.8: $\mathrm{M}_{1}(\cdot)$ is clearly an admissible mean function for an IIDOS/EOS model. If $\overline{\mathrm{L}}(\cdot)$ is the complementary mean function of the NHPP $\Lambda_{\sim}$, then the complementary mean function for the IIDOS $\Lambda$ is $\overline{\mathrm{L}}_{1}(\cdot)=([\mathrm{M}(\infty)+1 / 2] / \mathrm{M}(\alpha)) \overline{\mathrm{L}}(\cdot)=([\overline{\mathrm{L}}(0)+1 / 2] / \overline{\mathrm{L}}(0)) \overline{\mathrm{L}}(\cdot)$ 


$$
\begin{aligned}
& \text { and } \overline{\mathrm{L}}_{1}(\lambda)=\overline{\mathrm{L}}(\lambda)+\mathrm{c} \overline{\mathrm{L}}(\lambda) / \overline{\mathrm{L}}(0),|\mathrm{c}|<1 / 2 \text {, } \\
& \left|m(t)-m_{1}(t)\right|=\left|\int_{0}^{\infty} \lambda e^{-\lambda t}\left(d L(\lambda)-d L_{1}(\lambda)\right)\right| \\
& =|c| \int_{0}^{\infty} \lambda e^{-\lambda t} d \bar{L}(\lambda) / \bar{L}(0) \\
& \leq \frac{1}{2} \sup _{\lambda} \lambda e^{-\lambda t}=\frac{1}{2} \frac{1}{t} e^{-1}
\end{aligned}
$$

Proof of Theorem 6.9: $\quad \overline{\mathrm{L}}_{0}(\lambda)=[\overline{\mathrm{L}}(\lambda)+1 / 2]$ so $\left|\overline{\mathrm{L}}(\lambda)-\overline{\mathrm{L}}_{0}(\lambda)\right| \leq 1 / 2$. Two extreme cases are $\overline{\mathrm{L}}_{\mathrm{u}}(\lambda)=[\overline{\mathrm{L}}(\lambda)+1]$ and $\overline{\mathrm{L}}_{\ell}(\lambda)=[\overline{\mathrm{L}}(\lambda)]$, which satisfy

$$
\overline{\mathrm{L}}_{\mathbf{u}}(\lambda)>\overline{\mathrm{L}}(\lambda)>\overline{\mathrm{L}}_{\ell}(\lambda)
$$

and

$$
\overline{\mathrm{L}}_{\mathrm{u}}(\lambda)>\overline{\mathrm{L}}_{0}(\lambda)>\overline{\mathrm{L}}_{\ell}(\lambda)
$$

Since these are step functions and $1-e^{-\lambda t}$ is increasing in $\lambda$,

$$
M_{u}(t)=-\int\left(1-e^{-\lambda t}\right) d \bar{L}_{u}(\lambda)>M(t)>-\int\left(1-e^{-\lambda t}\right) d \bar{L}_{\ell}(\lambda)=M_{\ell}(t)
$$

and

$$
M_{u}(t)>M_{0}(t)>M_{\ell}(t)
$$

But

$$
M_{u}(t)-M_{\ell}(t)=\left(1-e^{-\lambda \cdot \infty}\right)-\left(1-e^{-\lambda \cdot 0}\right)=1 ;
$$

therefore $\left|M(t)-M_{0}(t)\right|<1,0<t<\infty$. Similarly, using the fact that $\lambda e^{-\lambda t}$ is unimodal in $\lambda$ with mode $1 / t$, one can bound it by the sum of two monotone functions:

$$
f_{1}(\lambda)=e^{-\lambda t} 1_{[0,1 / t]}(\lambda)+t^{-1} e^{-1} I_{[1 / t, \infty)}(\lambda)
$$


and

$$
f_{2}(\lambda)=t^{-1} e^{-1} 1_{[0,1 / t]}(\lambda)+\lambda e^{-\lambda t} 1_{[1 / t, \infty)}(\lambda)
$$

and use a combination of the above analyses to get

$$
\left|m(t)-m_{0}(t)\right|<\sup _{\lambda} f_{1}(\lambda)+\sup _{\lambda} f_{2}(\lambda)=2(t e)^{-1} .
$$

At the end of Section 6 the Geometric Order Statistic model is mentioned. This model might be preferred to an EOS model when the time parameter is integer-valued and corresponds to successive independent uses of the program. In this case the times until failure have a geometric distribution,

$$
\begin{aligned}
& P(X=t)=(1-p)^{t-1} p, \\
& P(X>t)=(1-p)^{t},
\end{aligned}
$$

$t=1,2,3, \ldots ; 0 \leq p \leq 1$, where $p$ is the failure probability on a given execution of the program. Denote the set of failure probabilities by $\underset{\sim}{p}=\left\{p_{1}, p_{2}, p_{3} \ldots\right\}$ and let this be a realization of a stochastic point process with complementary mean function $\overline{\mathrm{L}}(\cdot)$, i.e., $\overline{\mathrm{L}}(\mathrm{p})$ equals the number of bugs in the program with failure probabilities equal to or greater than $\mathrm{p}$. Let $\mathrm{x}_{1}, \mathrm{x}_{2}, \ldots$ be independent Geometric random variables with parameters $\mathrm{p}_{1}, \mathrm{p}_{2}, \ldots$, respectively. The failure process is

$$
N(t)=\sum_{i=1}^{\infty} 1_{[0, t]}\left(x_{i}\right), t=1,2,3, \ldots .
$$

For deterministic $\underset{\sim}{p}$, the mean function is

$$
\begin{aligned}
M(t)=\operatorname{EN}(t) & =\sum_{i=1}^{\infty} P\left(x_{i} \leq t\right) \\
& =\sum_{i=1}^{\infty}\left(1-\left(1-p_{i}\right)^{t}\right), t=1,2, \ldots,
\end{aligned}
$$

and its intensity is 


$$
\begin{aligned}
m(t) & =M(t)-M(t-1) \\
& =\sum_{i=1}^{\infty}\left(1-p_{i}\right)^{t-1} p_{i}, t=1,2, \ldots .
\end{aligned}
$$

In general

$$
\begin{aligned}
& M(t)=-\int_{0}^{1}\left(1-(1-p)^{t}\right) d \bar{L}(p), \\
& m(t)=-\int_{0}^{1}(1-p)^{t-1} p d \bar{L}(p)=-\int_{0}^{1}(1-p)^{t} \frac{p d \bar{L}(p)}{1-p} .
\end{aligned}
$$

Let us examine $m(t)$ : First note that $\left\{(1-p)^{t}, t=1,2,3, \ldots\right\}$ is a completely monotone sequence. Integration with respect to a positve measure preserves this property; therefore $\{m(t), t=1,2,3, \ldots\}$ is a completely monotone sequence. Second, consider

$$
\begin{aligned}
m(t) & =-\int_{0}^{1}(1-p)^{t} \frac{p d \bar{L}(p)}{1-p} \\
& =-\int_{0}^{1} \exp (t \log (1-p)) \frac{p d \bar{L}(p)}{1-p} \\
& =-\int_{0}^{1} \sum_{i=0}^{\infty}\left(\frac{t \log (1-p)}{i !}\right)^{i} \frac{p d \bar{L}(p)}{1-p} \\
& =\sum_{i=1}^{\infty} \frac{(-1)^{i} t^{i}}{i !} \int_{0}^{1}(-10 g(1-p))^{i} \frac{(-p d \bar{L}(p)}{1-p}
\end{aligned}
$$

Let

$$
c_{i}=\int_{0}^{1}(-\log (1-p))^{i}\left(\frac{-p d \bar{L}(p)}{1-p}\right), i=0,1,2, \ldots ;
$$

$\left\{c_{i} / c_{0}, i=0,1,2, \ldots\right\}$ is the moment sequence of the random variable $-\log (1-\mathrm{Q})$ where $\mathrm{P}(\mathrm{p}<\mathrm{Q}<\mathrm{p}+\mathrm{dp})=-\mathrm{c}_{0}^{-1} \mathrm{pd} \overline{\mathrm{L}}(\mathrm{p}) /(1-\mathrm{p})$. If $0<-\log (1-Q)<1$ then Theorem 6.10 applies and the moment sequence is completely monotone. 
But $0 \leq-\log (1-Q) \leq 1$ is equivalent to $0 \leq Q \leq 1-\mathrm{e}^{-1}$; thus a necessary and sufficient condition for a c.m. moment sequence is that the support of $-p d \bar{L}(p) / 1-p$ is contained in $\left[0,1-e^{-1}\right], i . e$. , $\overline{\mathrm{L}}(\mathrm{p})=0$ for $\mathrm{p}>1-\mathrm{e}^{-1}$. Thus we get qualitative properties for Geometric Order Statistic intensity sequences that are similar to EOS intensity functions except that completely monotone sequences take the place of completely monotone functions and in Theorem 6.11 the condition $0<\mathrm{p} \leq 1-\mathrm{e}^{-1}$ replaces $0<\lambda \leq 1$.

\section{Acknowledgment}

Mr. Mohammed Ahmed generated Figures 5.2 through 5.11. I thank him for his valuable contribution. 
[1] ADAMS, E. N. (1984). Optimizing preventive service of software products. IBM Journal of Research and Development, $28,2-14$.

[2] BILlingsleY, P. (1968). Convergence of Probability Measures. John Wiley \& Sons, Inc., New York.

[3] BREIMAN, L. (1969). Probability and Stochastic Processes, with a View Toward Applications. Houghton Mifflin Co., Boston.

[4] CAMPBELL, G. and K. O. OTT (1979). Statistical evaluation of major human errors during the development of new technological systems. Nuclear Science and Engineering, 71, 267-279.

[5] CINLAR, E. (1975). Introduction to Stochastic Processes, Englewood Cliffs, Prentice-Hall, Inc., New Jersey.

[6] CROW, L. H. (1974). Reliability analysis for complex, repairable systems. Reliability and Biometry (F. Proschan and R. J. Serfling, eds.) SIAM, Philadelphia. 379-410.

[7] DUANE, J. T. (1964). Learning curve approach to reliability monitoring. IEEE Transactions Aerospace, 2, 563-566.

[8] DUNHAM, J.R. and J. L. PIERCE (1984). An experiment in software reliability. Research Triangle Institute. NASA Contractor Report 172553, March 1985.

[9] FELLER, W. (1971). An introduction to Probability Theory and Its Applications - Vol. II, 2 Ed. John Wiley \& Sons, Inc., New York.

[10] GOEL, A. L. and K. OKUMOTO (1979). Time-dependent error detection rate model for software reliability and other performance measures. IEEE Transactions on Reliability, R-28, 206-211.

[11] HALSTEAD, M. H. (1977). Elements of Software Science. North-Holland Pub. Co., New York. 
[12] JELINSKI, Z. and P. B. MORANDA (1972). Software reliability research. Statistical Computer Performance Evaluation, (W. Freiberger, ed.), Academic Press, Inc., New York, 465-484.

[13] KARLIN, S. and H. M. TAYLOR (1981). A Second Course in Stochastic Processes, Academic Press, Inc., New York.

[14] LANGBERG, N. and N. D. SINGPURWALLA (1982). Unification of some software reliability models via the Bayesian approach, SIAM Journal of Scientific and Statistical Computing, Vol. 6 , No. 3 , 1985.

[15] LAW, A. M. and W. D. KELTON (1982). Simulation Modeling and Analysis, McGraw-Hill Book Co., New York.

[16] LITTLEWOOD, B. (1981). Stochastic reliability-growth: A model for fault-removal in computer-programs and hardware designs. IEEE Transactions on Reliability, $\mathrm{R}-30,313-320$.

[17] LITTLEWOOD, B. and J. L. VERRALL (1973). A Bayesian reliability growth model for computer software. Journal of the Royal Statistical Society Series (Applied Statistics), 22, 332-346.

[18] MILLER, D. R. (1976). Order statistics, poisson processes, and reparable systems. Journal Applied Probability, 13, 519-529.

[19] MILLER, D. R. and A. SOFER (1985). Completely monotone regression estimates of software failure rates. Institute for Management Science and Engineering, The George Washington University. Technical Paper Serial T-497.

[20] MUSA, J. D. and K. OKUMOTO (1984). A logarithmic poisson execution time model for software reliability measurement. Proceedings of the Seventh International Conference on Software Engineering. IEEE, New York, 230-238.

[21] NAGEL, P. M., F. W. SCHOLZ, and J. A. SKRIVAN (1984). Software reliability: additional investigations into modeling with replicated experiments. NASA CR-172378. 
[22] NAGEL, P. M. and J. A. SKRIVAN (1982). Software reliability: repetitive run experimentation and modeling. NASA CR-165836.

[23] ROSS, S. M. (1980). Introduction to Probability Models, 2 Ed. Academic Press, Inc., New York.

[24] ROSS, S. M. (1983). Stochastic Processes. John Wiley \& Sons, Inc., New York.

[25] SCHMEISER, B. W. (1978). The generation of order statistics in digital computer simulation: A survey. Proceedings of the 1978 Winter Simulation Conference. IEEE, New York, 137-140.

[26] SIMON, H. A. (1972). The sizes of things. Statistics: A Guide to the Unknown (J. M. Tanur, ed.), Holden-Day, Inc., San Francisco, 195-202. 


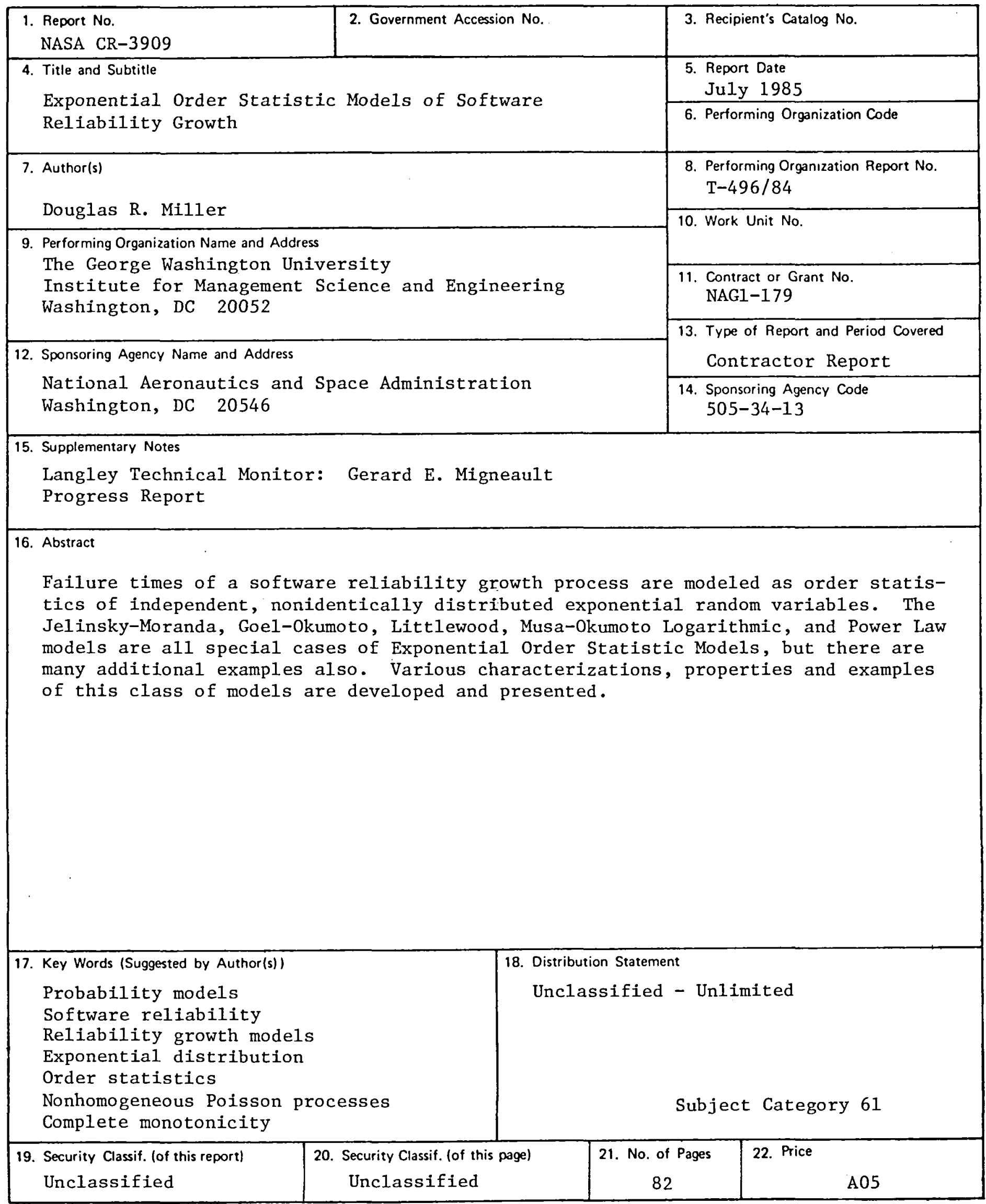


National Aeronautics and

Space Administration

Washington, D.C.

20546

Official Business

BULK RATE

POSTAGE \& FEES PAID

NASA Washington, DC

Permit No. G-27

Penalty for Private Use, $\$ 300$ 